\title{
SYMPLECTIC JACOBI DIAGRAMS AND THE LIE ALGEBRA OF HOMOLOGY CYLINDERS
}

\author{
KAZUO HABIRO AND GWÉNAËL MASSUYEAU
}

\begin{abstract}
Let $S$ be a compact connected oriented surface, whose boundary is connected or empty. A homology cylinder over the surface $S$ is a cobordism between $S$ and itself, homologically equivalent to the cylinder over $S$. The $Y$-filtration on the monoid of homology cylinders over $S$ is defined by clasper surgery. Using a functorial extension of the Le-Murakami-Ohtsuki invariant, we show that the graded Lie algebra associated to the $Y$-filtration is isomorphic to the Lie algebra of "symplectic Jacobi diagrams". This Lie algebra consists of the primitive elements of a certain Hopf algebra whose multiplication is a diagrammatic analogue of the Moyal-Weyl product.

The mapping cylinder construction embeds the Torelli group into the monoid of homology cylinders, sending the lower central series to the $Y$-filtration. We give a combinatorial description of the graded Lie algebra map induced by this embedding, by connecting Hain's infinitesimal presentation of the Torelli group to the Lie algebra of symplectic Jacobi diagrams. This Lie algebra map is shown to be injective in degree two, and the question of the injectivity in higher degrees is discussed.
\end{abstract}

\section{Contents}

1. Introduction and statement of the results

2. Diagrammatic description of the Lie algebra of homology cylinders 6

3. The Lie algebra of symplectic Jacobi diagrams 10

$\begin{array}{ll}\text { 4. Algebraic description of the mapping cylinder construction } & 15\end{array}$

5. The degree two case 17

6. Stability with respect to the genus 22

7. The closed surface case 23

8. Remarks and questions 33

Appendix A. On Malcev Lie algebras of filtered groups 36

References 40

\section{Introduction AND STATEMENT OF the RESUlts}

Let $\Sigma_{g, 1}$ be a compact connected oriented surface of genus $g$ with one boundary component. The first homology group $H_{1}\left(\Sigma_{g, 1} ; \mathbb{Z}\right)$ is denoted by $H$ and is equipped with the intersection pairing

$$
\omega: H \otimes H \longrightarrow \mathbb{Z} .
$$

This is a non-degenerate skew-symmetric form, the group of isometries of which is denoted by $\operatorname{Sp}(H)$. Similarly, $H_{\mathbb{Q}}:=H \otimes \mathbb{Q}$ is equipped with the rational extension of $\omega$ and $\operatorname{Sp}\left(H_{\mathbb{Q}}\right)$ denotes the group of isometries of the symplectic vector space $H_{\mathbb{Q}}$.

Date: November 30, 2007.

2000 Mathematics Subject Classification. 57M27, 57R50, 20F38, $20 \mathrm{~F} 40$.

Key words and phrases. 3-manifold, monoid of homology cylinders, Torelli group, finite-type invariant, Jacobi diagram, clasper, LMO invariant, Malcev completion, Malcev Lie algebra.

The first author is partially supported by Grant-in-Aid for Scientific Research (C) 19540077. 
1.1. The Torelli group. Let $\mathcal{I}_{g, 1}$ be the Torelli group of $\Sigma_{g, 1}$, which is the subgroup of the mapping class group $\mathcal{M}_{g, 1}$ of $\Sigma_{g, 1}$ consisting of the elements acting trivially on homology. A good introduction to the Torelli group is found in Johnson's survey [17].

Commutator calculus is one of the most important tools in the study of the Torelli group. The group $\mathcal{I}_{g, 1}$ is filtered by its lower central series

$$
\mathcal{I}_{g, 1}=\Gamma_{1} \mathcal{I}_{g, 1} \supset \Gamma_{2} \mathcal{I}_{g, 1} \supset \Gamma_{3} \mathcal{I}_{g, 1} \supset \cdots .
$$

The pronilpotent completion of $\mathcal{I}_{g, 1}$ is

$$
\widehat{\mathcal{I}}_{g, 1}:={\underset{\varliminf}{\leftarrow}}_{i} \mathcal{I}_{g, 1} / \Gamma_{i} \mathcal{I}_{g, 1}
$$

and the canonical map $\mathcal{I}_{g, 1} \rightarrow \widehat{\mathcal{I}}_{g, 1}$ is injective. The graded Lie algebra over $\mathbb{Z}$ associated to the lower central series of $\mathcal{I}_{g, 1}$, namely

$$
\operatorname{Gr}^{\Gamma} \mathcal{I}_{g, 1}=\bigoplus_{i \geq 1} \operatorname{Gr}_{i}^{\Gamma} \mathcal{I}_{g, 1}, \quad \text { where } \operatorname{Gr}_{i}^{\Gamma} \mathcal{I}_{g, 1}:=\Gamma_{i} \mathcal{I}_{g, 1} / \Gamma_{i+1} \mathcal{I}_{g, 1}
$$

is called the Torelli Lie algebra of $\Sigma_{g, 1}$. With rational coefficients, the Torelli Lie algebra

$$
\operatorname{Gr}^{\Gamma} \mathcal{I}_{g, 1} \otimes \mathbb{Q}
$$

is, by a general fact, canonically isomorphic to the graded Lie algebra associated to the complete lower central series of the Malcev Lie algebra of $\mathcal{I}_{g, 1}$.

The Torelli Lie algebra with rational coefficients is generated by its degree 1 part $\operatorname{Gr}_{1}^{\Gamma} \mathcal{I}_{g, 1} \otimes \mathbb{Q}$. If $g \geq 3$, this vector space can be identified with $\Lambda^{3} H_{\mathbb{Q}}$ by extending the first Johnson homomorphism

$$
\tau_{1}: \mathcal{I}_{g, 1} \longrightarrow \Lambda^{3} H
$$

to rational coefficients [16]. Hence a Lie algebra epimorphism

$$
J: \operatorname{Lie}\left(\Lambda^{3} H_{\mathbb{Q}}\right) \longrightarrow \operatorname{Gr}^{\Gamma} \mathcal{I}_{g, 1} \otimes \mathbb{Q},
$$

where $\operatorname{Lie}\left(\Lambda^{3} H_{\mathbb{Q}}\right)$ is the free Lie algebra over $\Lambda^{3} H_{\mathbb{Q}}$. The ideal of relations of the Torelli Lie algebra is

$$
\mathrm{R}\left(\mathcal{I}_{g, 1}\right)=\bigoplus_{i \geq 1} \mathrm{R}_{i}\left(\mathcal{I}_{g, 1}\right):=\operatorname{ker}(J)
$$

Let $\bar{J}: \operatorname{Lie}\left(\Lambda^{3} H_{\mathbb{Q}}\right) / \mathrm{R}\left(\mathcal{I}_{g, 1}\right) \rightarrow \operatorname{Gr}^{\Gamma} \mathcal{I}_{g, 1} \otimes \mathbb{Q}$ be the isomorphism induced by $J$. The following theorem is proved by Hain in [14].

Theorem 1.1 (Hain). If $g \geq 3$, then the Malcev Lie algebra of $\mathcal{I}_{g, 1}$ is isomorphic to the completion of $\mathrm{Gr}^{\Gamma} \mathcal{I}_{g, 1} \otimes \mathbb{Q}$. Moreover, the ideal $\mathrm{R}\left(\mathcal{I}_{g, 1}\right)$ is generated by $\mathrm{R}_{2}\left(\mathcal{I}_{g, 1}\right)$ for $g \geq 6$, and by $\mathrm{R}_{2}\left(\mathcal{I}_{g, 1}\right)+\mathrm{R}_{3}\left(\mathcal{I}_{g, 1}\right)$ for $3 \leq g \leq 5$.

The first half of Theorem 1.1 implies that the Torelli Lie algebra has all the information about the Malcev completion of $\mathcal{I}_{g, 1}$. The second half implies that one obtains a presentation of the Torelli Lie algebra by computing the quadratic/cubic relations (see $[14,12]$ for $g \geq 6)$. 
1.2. The monoid of homology cylinders. Homology cylinders over $\Sigma_{g, 1}$ are cobordisms from $\Sigma_{g, 1}$ to itself with the same homology type as the cylinder over $\Sigma_{g, 1}$. The set $\mathcal{C}_{g, 1}$ of homeomorphism types (relative to boundary parameterization) of homology cylinders is a monoid, with multiplication being the usual pasting operation of cobordisms. Homology cylinders are introduced in [9, 13], see also [22, 8, 11, 25, 36, 24].

Calculus of claspers $[10,13]$ works as "topological commutator calculus" on the monoid $\mathcal{C}_{g, 1}$, where the usual algebraic commutator calculus does not work. The role of the lower central series is played by the family of the $Y_{i}$-equivalence relations. For each $i \geq 1$, the $Y_{i}$-equivalence on homology cylinders is generated by surgeries along $Y_{i}$-claspers, which are connected graph claspers with $i$ nodes. The $Y_{i}$-equivalence is also generated by Torelli surgeries of class $i$, i.e. surgeries along an embedding of the surface $\Sigma_{h, 1}$ with any $h \geq 0$ using any element of $\Gamma_{i} \mathcal{I}_{h, 1}$ (see $\left.[13,24]\right)$. The $Y_{i}$-equivalence becomes finer as $i$ increases.

For each $i \geq 1$, the quotient monoid $\mathcal{C}_{g, 1} / Y_{i}$ is a finitely generated, nilpotent group, and there is a sequence of surjective homomorphisms

$$
\cdots \longrightarrow \mathcal{C}_{g, 1} / Y_{3} \longrightarrow \mathcal{C}_{g, 1} / Y_{2} \longrightarrow \mathcal{C}_{g, 1} / Y_{1}=\{1\} .
$$

The completion

$$
\widehat{\mathcal{C}}_{g, 1}:=\lim _{i} \mathcal{C}_{g, 1} / Y_{i}
$$

is called the group of homology cylinders ${ }^{1}$. Conjecturally, the canonical homomorphism $\mathcal{C}_{g, 1} \rightarrow \widehat{\mathcal{C}}_{g, 1}$ is injective.

Denoting by $Y_{i} \mathcal{C}_{g, 1}$ the submonoid of $\mathcal{C}_{g, 1}$ consisting of homology cylinders which are $Y_{i}$-equivalent to the trivial cylinder, one obtains the $Y$-filtration

$$
\mathcal{C}_{g, 1}=Y_{1} \mathcal{C}_{g, 1} \supset Y_{2} \mathcal{C}_{g, 1} \supset Y_{3} \mathcal{C}_{g, 1} \supset \cdots
$$

for the monoid $\mathcal{C}_{g, 1}$. The quotient monoid $Y_{k} \mathcal{C}_{g, 1} / Y_{l}$ is a subgroup of $\mathcal{C}_{g, 1} / Y_{l}$ for all $l \geq k \geq 1$ and, furthermore, the inclusion

$$
\left[Y_{j} \mathcal{C}_{g, 1} / Y_{l}, Y_{k} \mathcal{C}_{g, 1} / Y_{l}\right] \subset Y_{j+k} \mathcal{C}_{g, 1} / Y_{l}
$$

is satisfied for all $j, k \geq 1$ and $l \geq j+k$. Thus, there is a graded Lie algebra over $\mathbb{Z}$

$$
\operatorname{Gr}^{Y} \mathcal{C}_{g, 1}:=\bigoplus_{i \geq 1} Y_{i} \mathcal{C}_{g, 1} / Y_{i+1}
$$

which we call the Lie algebra of homology cylinders.

As proposed in [13] and established in [4], there is a diagrammatic version of the Lie algebra $\operatorname{Gr}^{Y} \mathcal{C}_{g, 1} \otimes \mathbb{Q}$. Similar diagrammatic constructions have also been considered by Garoufalidis and Levine [8] and by Habegger [11]. Our diagrammatic description of $\operatorname{Gr}^{Y} \mathcal{C}_{g, 1} \otimes \mathbb{Q}$ involves a graded Lie algebra of Jacobi diagrams

$$
\mathcal{A}^{<, c}\left(H_{\mathbb{Q}}\right)=\bigoplus_{i \geq 1} \mathcal{A}_{i}^{<, c}\left(H_{\mathbb{Q}}\right) .
$$

Here, the vector space $\mathcal{A}_{i}^{<, c}\left(H_{\mathbb{Q}}\right)$ is spanned by connected Jacobi diagrams with $i$ internal vertices and with external vertices totally ordered and labeled by elements of $H_{\mathbb{Q}}$, modulo the AS, IHX, STU-like and multilinearity relations. The following theorem is essentially proved in [4], see $\S 2.3$.

\footnotetext{
${ }^{1}$ The group $\widehat{\mathcal{C}}_{g, 1}$ is different from the homology cobordism group of homology cylinders introduced by Levine [22], which is a non-trivial quotient of $\mathcal{C}_{g, 1}$.
} 
Theorem 1.2. For $g \geq 0$, there are graded Lie algebra isomorphisms

$$
\mathcal{A}^{<, c}\left(H_{\mathbb{Q}}\right) \underset{\mathrm{LMO}}{\rightleftarrows} \operatorname{Gr}^{Y} \mathcal{C}_{g, 1} \otimes \mathbb{Q},
$$

which are inverse to each other.

The isomorphism $\psi$ is a "surgery" map sending each Jacobi diagram to surgery along its corresponding graph clasper in the trivial cylinder over $\Sigma_{g, 1}$. The isomorphism LMO comes from a functorial version of the Le-Murakami-Ohtsuki invariant $[21,2]$ constructed in [4].

The $Y$-filtration (1.1) on $\mathcal{C}_{g, 1}$ induces a similar filtration on the group $\widehat{\mathcal{C}}_{g, 1}$

$$
\widehat{\mathcal{C}}_{g, 1}=\widehat{Y}_{1} \widehat{\mathcal{C}}_{g, 1} \supset \widehat{Y}_{2} \widehat{\mathcal{C}}_{g, 1} \supset \widehat{Y}_{3} \widehat{\mathcal{C}}_{g, 1} \supset \cdots
$$

defined by

$$
\widehat{Y}_{j} \widehat{\mathcal{C}}_{g, 1}:=\lim _{i \geq j} Y_{j} \mathcal{C}_{g, 1} / Y_{i}
$$

In the appendix, we define the Malcev Lie algebra of a filtered group, which corresponds to the usual notion of Malcev Lie algebra when the group is filtered by the lower central series. We also extend to this setting some well-known properties of Malcev Lie algebras and Malcev completions. In particular, we consider in $\S 2.4$ the Malcev Lie algebra of the group $\widehat{\mathcal{C}}_{g, 1}$ endowed with the $\widehat{Y}$-filtration and prove it to be isomorphic to the completion of $\mathcal{A}^{<, c}\left(H_{\mathbb{Q}}\right)$. This enhances Theorem 1.2 since, by a general fact, the graded Lie algebra associated to a filtered group is canonically isomorphic to the graded Lie algebra associated to the canonical filtration on its Malcev Lie algebra.

In $\S 3$ we give an alternative description of the Lie algebra $\mathcal{A}^{<, c}\left(H_{\mathbb{Q}}\right)$. For this, we consider the graded vector space $\mathcal{A}^{c}\left(H_{\mathbb{Q}}\right)$ spanned by connected Jacobi diagrams with external vertices labeled by elements of $H_{\mathbb{Q}}$, subject to the AS, IHX and multilinearity relations. There is no ordering of the external vertices anymore. We define a Lie algebra structure on $\mathcal{A}^{c}\left(H_{\mathbb{Q}}\right)$, which is isomorphic to that of $\mathcal{A}^{<, c}\left(H_{\mathbb{Q}}\right)$ via a "symmetrization" map

$$
\chi: \mathcal{A}^{c}\left(H_{\mathbb{Q}}\right) \stackrel{\simeq}{\longrightarrow} \mathcal{A}^{<, c}\left(H_{\mathbb{Q}}\right) .
$$

The Lie algebra $\mathcal{A}^{c}\left(H_{\mathbb{Q}}\right)$ consists of the primitive elements of a Hopf algebra $\mathcal{A}\left(H_{\mathbb{Q}}\right)$ of "symplectic Jacobi diagrams", whose associative multiplication is a diagrammatic analogue of the Moyal-Weyl product. This analogy is justified by considering weight systems associated to metrized Lie algebras.

1.3. The mapping cylinder construction. As proposed by the first author in [13], the injective monoid homomorphism

$$
\text { c: } \mathcal{I}_{g, 1} \hookrightarrow \mathcal{C}_{g, 1}
$$

defined by the mapping cylinder construction serves as a useful tool in the study of the Torelli group. It follows from the inclusion (1.2) that $\mathbf{c}$ sends the lower central series of $\mathcal{I}_{g, 1}$ to the $Y$-filtration of $\mathcal{C}_{g, 1}$ :

$$
\mathbf{c}\left(\Gamma_{i} \mathcal{I}_{g, 1}\right) \subset Y_{i} \mathcal{C}_{g, 1} \quad \text { for all } i \geq 1 .
$$

So, $\mathbf{c}$ induces a group homomorphism

$$
\widehat{\mathbf{c}}: \widehat{\mathcal{I}}_{g, 1} \longrightarrow \widehat{\mathcal{C}}_{g, 1},
$$


as well as a graded Lie algebra homomorphism

Grc: $\operatorname{Gr}^{\Gamma} \mathcal{I}_{g, 1} \longrightarrow \operatorname{Gr}^{Y} \mathcal{C}_{g, 1}$.

It is natural to ask whether the homomorphisms $\widehat{\mathbf{c}}$ and $\operatorname{Gr} \mathbf{c}$ are injective or not. For example, in degree 1 , the homomorphism

$$
\mathrm{Gr}_{1} \mathbf{c}: \mathcal{I}_{g, 1} /\left[\mathcal{I}_{g, 1}, \mathcal{I}_{g, 1}\right] \longrightarrow \mathcal{C}_{g, 1} / Y_{2}
$$

is an isomorphism for $g \geq 3[13,25]$.

Question 1.3. Is the graded Lie algebra homomorphism $\operatorname{Grc}: \operatorname{Gr}^{\Gamma} \mathcal{I}_{g, 1} \rightarrow \operatorname{Gr}^{Y} \mathcal{C}_{g, 1}$ injective when $g \geq 3$ ?

If $g=2$, Gr $\mathbf{c}$ is certainly not injective because $\operatorname{Gr}_{1}^{\Gamma} \mathcal{I}_{2,1}$ is not finitely generated ${ }^{2}$.

The question has been asked by the first author in [13] to clarify the relationships between Hain's presentation of the Torelli Lie algebra and diagrammatic descriptions of the Lie algebra of homology cylinders. The following result is a starting point of studies in this direction.

Theorem 1.4. If $g \geq 3$, then the following diagram in the category of graded Lie algebras with $\operatorname{Sp}\left(H_{\mathbb{Q}}\right)$-actions is commutative:

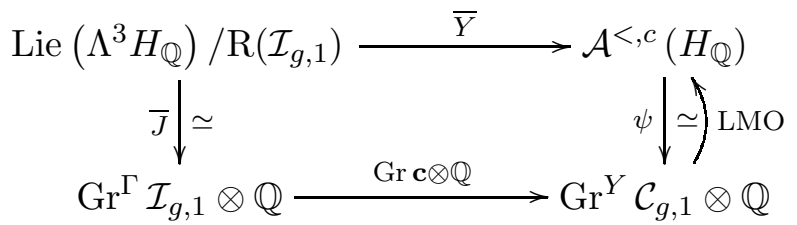

Here, $\bar{Y}$ is induced by the Lie algebra homomorphism $Y: \operatorname{Lie}\left(\Lambda^{3} H_{\mathbb{Q}}\right) \rightarrow \mathcal{A}^{<, c}\left(H_{\mathbb{Q}}\right)$ which, in degree 1 , sends the trivector $x \wedge y \wedge z$ to the $Y$-graph $x y z$.

Thus, Theorem 1.4 reduces the study of the map $\operatorname{Gr} \mathbf{c} \otimes \mathbb{Q}$ to the understanding of the algebraically-defined map

$$
\bar{Y}: \operatorname{Lie}\left(\Lambda^{3} H_{\mathbb{Q}}\right) / \mathrm{R}\left(\mathcal{I}_{g, 1}\right) \longrightarrow \mathcal{A}^{<, c}\left(H_{\mathbb{Q}}\right),
$$

the source of which is described by Hain's result (Theorem 1.1). Theorem 1.4 is proved in $\S 4$, where the symplectic actions in diagram (1.7) are also specified.

In $\S 5$ we use the Lie algebra $\mathcal{A}^{c}\left(H_{\mathbb{Q}}\right)$ to compute the Lie bracket of $\mathcal{A}^{<, c}\left(H_{\mathbb{Q}}\right)$ in degree $1+1$. Thus, we obtain the following result:

Theorem 1.5. If $g \geq 3$, then the kernel of $Y_{2}: \operatorname{Lie}_{2}\left(\Lambda^{3} H_{\mathbb{Q}}\right) \rightarrow \mathcal{A}_{2}^{<, c}\left(H_{\mathbb{Q}}\right)$ coincides with the submodule $\mathrm{R}_{2}\left(\mathcal{I}_{g, 1}\right)$.

This can be regarded as a diagrammatic formulation of prior results by Morita [27, 28] and Hain [14], so that some computations done in $\S 5$ to prove it should be essentially well-known to experts. We also identify the image of $\chi_{2}^{-1} \circ Y_{2}: \operatorname{Lie}_{2}\left(\Lambda^{3} H_{\mathbb{Q}}\right) \rightarrow \mathcal{A}_{2}^{c}\left(H_{\mathbb{Q}}\right)$ with the even part $\mathcal{A}_{2, \mathrm{ev}}^{c}\left(H_{\mathbb{Q}}\right)$, consisting of Jacobi diagrams whose first Betti number is even.

Theorems 1.4 and 1.5 give a partial answer to Question 1.3 in degree 2:

Corollary 1.6. If $g \geq 3$, then the map $\operatorname{Gr}_{2} \mathbf{c} \otimes \mathbb{Q}: \operatorname{Gr}_{2}^{\Gamma} \mathcal{I}_{g, 1} \otimes \mathbb{Q} \longrightarrow \operatorname{Gr}_{2}^{Y} \mathcal{C}_{g, 1} \otimes \mathbb{Q}$ is injective.

\footnotetext{
${ }^{2}$ Indeed, the group $\mathcal{I}_{2,1}$ is free of infinite rank [26].
} 
There is also a "stabilized" form of Question 1.3.

Conjecture 1.7. For $g \geq 3$, the map

$$
\underset{g}{\lim } \operatorname{Gr} \mathbf{c}: \underset{g}{\lim } \operatorname{Gr}^{\Gamma} \mathcal{I}_{g, 1} \longrightarrow \underset{g}{\lim } \operatorname{Gr}^{Y} \mathcal{C}_{g, 1}
$$

is injective, where the spaces and the map are induced by a sequence of surface inclusions $\Sigma_{0,1} \subset \Sigma_{1,1} \subset \Sigma_{2,1} \subset \cdots$.

Conjecture 1.7 is equivalent to the conjecture stated in [13] that the lower central series of $\mathcal{I}_{g, 1}$ and the restriction to $\mathcal{I}_{g, 1}$ of the $Y$-filtration of $\mathcal{C}_{g, 1}$ are stably equal. Some stability properties are discussed in $\S 6$.

The case of a closed connected oriented surface $\Sigma_{g}$ of genus $g$ is considered in $\S 7$, where analogues of Theorems 1.2, 1.4 and 1.5 are proved.

The final $\S 8$ concludes with further problems and remarks. The problems of determining the kernel and the image of the map $Y$ in higher degree are discussed, and such problems are related to questions about Johnson homomorphisms.

\section{Diagrammatic Description of the Lie AlgeBRa of HOMOlOGy Cylinders}

In this section, we recall from $[13,4]$ the main ingredients to obtain Theorem 1.2, which gives a diagrammatic description of the Lie algebra of homology cylinders. Furthermore, we produce from the LMO invariant a diagrammatric description of the Malcev Lie algebra of the group of homology cylinders.

2.1. The algebra $\mathcal{A}^{<}\left(H_{\mathbb{Q}}\right)$ and the Lie algebra $\mathcal{A}^{<, c}\left(H_{\mathbb{Q}}\right)$. First of all, we recall the definition of the cocommutative Hopf algebra $\mathcal{A}^{<}\left(H_{\mathbb{Q}}\right)$, which was introduced in [13] and used in [4].

A Jacobi diagram is a finite graph whose vertices have valence 1 (external vertices) or 3 (internal vertices). Each internal vertex is oriented, in the sense that its incident edges are cyclically ordered. A Jacobi diagram is colored by a set $S$ if a map from the set of its external vertices to $S$ is specified. A strut is a Jacobi diagram with only two external vertices and no internal vertex. The internal degree of a Jacobi diagram is the number of its internal vertices.

We define the following $\mathbb{Q}$-vector space

$$
\mathcal{A}^{<}\left(H_{\mathbb{Q}}\right):=\frac{\mathbb{Q} \cdot\left\{\begin{array}{l}
\text { Jacobi diagrams without strut component and with } \\
\text { external vertices totally ordered and colored by } H_{\mathbb{Q}}
\end{array}\right\}}{\text { AS, IHX, STU-like, multilinearity }}
$$

which is also denoted simply by $\mathcal{A}^{<}$. Here, the AS and IHX relations among Jacobi diagrams are the usual ones, namely<smiles>CC(C)(C)C1=[13C](I)C1(C)C</smiles>

AS

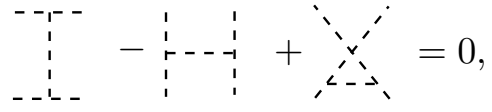

IHX

and the STU-like and multilinearity relations are defined by 


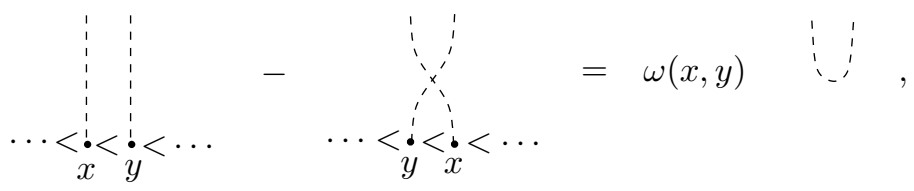

$\begin{array}{r}\vdots \\ \vdots \\ \vdots \\ \vdots+y\end{array}=\begin{aligned} & 1 \\ & \vdots \\ & \vdots \\ & \vdots\end{aligned} \quad+\quad \begin{gathered}1 \\ \vdots\end{gathered}$

The space $\mathcal{A}^{<}\left(H_{\mathbb{Q}}\right)$ is graded by the internal degree of Jacobi diagrams. Its degree completion will also be denoted by $\mathcal{A}^{<}\left(H_{\mathbb{Q}}\right)$.

There is also a space $\mathcal{A}^{<}\left(-H_{\mathbb{Q}}\right)$ defined as $\mathcal{A}^{<}\left(H_{\mathbb{Q}}\right)$ except that one uses the symplectic form $-\omega$ in the STU-like relation instead of $\omega$. There is a canonical isomorphism

$$
s: \mathcal{A}^{<}\left(-H_{\mathbb{Q}}\right) \longrightarrow \mathcal{A}^{<}\left(H_{\mathbb{Q}}\right)
$$

defined by $s(D)=(-1)^{\chi(D)} D$ for any Jacobi diagram $D$ with Euler characteristic $\chi(D)$.

Remark 2.1. Note that $\mathcal{A}^{<}\left(H_{\mathbb{Q}}\right)$ depends not only on the vector space $H_{\mathbb{Q}}$ but also on the symplectic form $\omega$, with which $H_{\mathbb{Q}}$ is implicitly equipped. The space $\mathcal{A}^{<}\left(H_{\mathbb{Q}}\right)$ is denoted by $\mathcal{A}\left(\Sigma_{g, 1}\right)$ in [13], while $\mathcal{A}^{<}\left(-H_{\mathbb{Q}}\right)$ corresponds to the space $\mathcal{A}\left(\Sigma_{g, 1}\right)$ in [4].

The multiplication $D_{\sqcup}^{<} E$ of two Jacobi diagrams $D, E \in \mathcal{A}^{<}$is the disjoint union of $D$ and $E$, the external vertices of $E$ being considered as "larger" than those of $D$. Then, $\mathcal{A}^{<}$is an associative algebra whose unit element is the empty diagram.

Like many other algebras of Jacobi diagrams in the literature, the algebra $\mathcal{A}^{<}$has a structure of a cocommutative Hopf algebra [4]. The comultiplication $\Delta: \mathcal{A}^{<} \rightarrow \mathcal{A}^{<} \otimes \mathcal{A}^{<}$ for a Jacobi diagram $D \in \mathcal{A}^{<}$is defined by

$$
\Delta(D)=\sum_{D=D^{\prime} \sqcup D^{\prime \prime}} D^{\prime} \otimes D^{\prime \prime},
$$

where the sum is over all the decompositions of $D$ into two families of connected components $D^{\prime}, D^{\prime \prime}$; in the right hand side, the orders of the external vertices of $D^{\prime}$ and $D^{\prime \prime}$ are induced by that of $D$. The counit $\varepsilon: \mathcal{A}^{<} \rightarrow \mathbb{Q}$ for a diagram $D \in \mathcal{A}^{<}$is defined by

$$
\varepsilon(D)= \begin{cases}1 & \text { if } D \text { is empty } \\ 0 & \text { otherwise }\end{cases}
$$

The antipode $S: \mathcal{A}^{<} \rightarrow \mathcal{A}^{<}$is the unique algebra anti-automorphism satisfying $S(D)=$ $-D$ for each non-empty connected Jacobi diagram $D \in \mathcal{A}^{<}$.

As is well known, the set of primitive elements $\mathrm{P}(A)$ in a Hopf algebra $A$ forms a Lie algebra, with the Lie bracket given by $[x, y]=x y-y x$. Thus, we have the Lie algebra $\mathrm{P}\left(\mathcal{A}^{<}\right)$of primitives in $\mathcal{A}^{<}$. Moreover, the Hopf algebra $\mathcal{A}^{<}$being cocommutative, the Milnor-Moore theorem asserts that $\mathcal{A}^{<}$is canonically isomorphic to the universal enveloping algebra $U \mathrm{P}\left(\mathcal{A}^{<}\right)$of $\mathrm{P}\left(\mathcal{A}^{<}\right)$.

Let $\mathcal{A}^{<, c}\left(H_{\mathbb{Q}}\right)$ (or simply $\left.\mathcal{A}^{<, c}\right)$ denote the subspace of $\mathcal{A}^{c}\left(H_{\mathbb{Q}}\right)$ spanned by the connected Jacobi diagrams.

Lemma 2.2. We have $\mathcal{A}^{<, c}=\mathrm{P}\left(\mathcal{A}^{<}\right)$.

Proof. Clearly, connected Jacobi diagrams are primitive. Thus we have $\mathcal{A}^{<, c} \subset \mathrm{P}\left(\mathcal{A}^{<}\right)$. Using the STU-like relation, one can check that $\mathcal{A}^{<, c}$ is a Lie subalgebra of $\mathrm{P}\left(\mathcal{A}^{<}\right)$and that the algebra $\mathcal{A}^{<}$is generated by $\mathcal{A}^{<, c}$. Since $\mathcal{A}^{<}=U \mathrm{P}\left(\mathcal{A}^{<}\right)$, it follows from the Poincaré-Birkhoff-Witt theorem that $\mathcal{A}^{<, c}=\mathrm{P}\left(\mathcal{A}^{<}\right)$. 
The natural $\operatorname{Sp}\left(H_{\mathbb{Q}}\right)$-action on $H_{\mathbb{Q}}$ induces an $\operatorname{Sp}\left(H_{\mathbb{Q}}\right)$-action on $\mathcal{A}^{<}\left(H_{\mathbb{Q}}\right)$, which is easily seen to be compatible with the Hopf algebra structure. In particular, $\mathcal{A}^{<, c}\left(H_{\mathbb{Q}}\right)$ is equipped with an $\operatorname{Sp}\left(H_{\mathbb{Q}}\right)$-action compatible with the Lie algebra structure.

2.2. The surgery map $\psi$. As suggested in [13], there is a canonical linear isomorphism

$$
\psi: \mathcal{A}^{<, c} \longrightarrow \operatorname{Gr}^{Y} \mathcal{C}_{g, 1} \otimes \mathbb{Q}
$$

defined by mapping each connected Jacobi diagram $D$ to the 3-manifold obtained from the cylinder $\Sigma_{g, 1} \times[-1,1]$ by surgery along a graph clasper $C(D)$ obtained from $D$ as follows:

- Thicken $D$ to an oriented surface using the vertex-orientation of $D$ (vertices are thickened to disks, and edges to bands). Cut a smaller disk in the interior of each disk that has been produced from an external vertex of $D$. This leads to an oriented compact surface $S(D)$, decomposed between disks, bands and annuli (corresponding to internal vertices, edges and external vertices of $D$ respectively). Use the induced orientation on $\partial S(D)$ to orient the cores of the annuli.

- Next, embed $S(D)$ into the interior of $\Sigma_{g, 1} \times[-1,1]$ in such a way that each annulus of $S(D)$ represents in $H_{\mathbb{Q}}$ the color of the corresponding external vertex of $D$. Moreover, the annuli should be in disjoint "horizontal slices" of $\Sigma_{g, 1} \times$ $[-1,1]$ and their "vertical height" along $[-1,1]$ should respect the total ordering of the external vertices of $D$. Such an embedding defines a graph clasper $C(D)$ in $\Sigma_{g, 1} \times[-1,1]$.

That $\psi$ is well-defined and surjective follows from clasper calculus $[13,10,7]$ : For the detail of the degree 1 case, see [25]; the higher degree case, where one has to consider also the IHX relation, is similar and needs the zip construction [13]. Using clasper calculus, one can also check that $\psi$ is a Lie algebra homomorphism. See also [8] and [11] for similar constructions.

To prove the injectivity of $\psi$, one needs the LMO invariant.

2.3. The LMO map. In a joint work with Cheptea [4], the authors extended the LMO invariant of homology 3-spheres to a functor on a category of Lagrangian cobordisms, which are cobordisms between surfaces with connected boundary, satisfying certain homological conditions. (Some extensions of the LMO invariant to cobordisms were previously constructed by Murakami and Ohtsuki [31] and by Cheptea and Le [5].) Since homology cylinders over $\Sigma_{g, 1}$ are Lagrangian cobordisms, the LMO functor restricts to a monoid homomorphism

$$
\widetilde{Z}^{Y}: \mathcal{C}_{g, 1} \longrightarrow \mathcal{A}^{Y}\left(\lfloor g\rceil^{+} \cup\lfloor g\rceil^{-}\right)
$$

with values in a certain complete Hopf algebra of Jacobi diagrams. The latter is isomorphic via a certain map $\varphi$ defined in [4] to $\mathcal{A}^{<}\left(-H_{\mathbb{Q}}\right)$. Thus, the composition $s \circ \varphi \circ \widetilde{Z}^{Y}$ defines a monoid homomorphism

$$
\mathcal{C}_{g, 1} \longrightarrow \mathcal{A}^{<}\left(H_{\mathbb{Q}}\right)
$$

Since $\widetilde{Z}^{Y}$ is an isomorphism at the level of graded Lie algebras [4], the monoid homomorphism (2.1) induces a graded Lie algebra isomorphism

$$
\text { LMO: } \operatorname{Gr}^{Y} \mathcal{C}_{g, 1} \otimes \mathbb{Q} \stackrel{\simeq}{\longrightarrow} \mathcal{A}^{<, c}\left(H_{\mathbb{Q}}\right) \text {. }
$$

Taking care of signs, we also deduce from [4] that $\psi$ and LMO are inverse to each other. Thus we have Theorem 1.2. 
2.4. The Malcev Lie algebra of the group of homology cylinders. The LMO functor can be used to prove more than Theorem 1.2: It also produces a diagrammatic description of the Malcev Lie algebra of $\widehat{\mathcal{C}}_{g, 1}$. Here, the Malcev Lie algebra is defined with respect to the $\widehat{Y}$-filtration (1.4) rather than the lower central series of $\widehat{\mathcal{C}}_{g, 1}$, the former being more natural than the latter from the point of view of finite-type invariants. Malcev completions and Malcev Lie algebras of filtered groups are presented in the appendix.

To deal with the Malcev Lie algebra of $\widehat{\mathcal{C}}_{g, 1}$, we come back to the monoid homomorphism (2.1), which we also denote by LMO:

$$
\mathrm{LMO}: \mathcal{C}_{g, 1} \longrightarrow \mathcal{A}^{<}
$$

It is shown in [4] that, if an $M \in \mathcal{C}_{g, 1}$ is $Y_{i}$-equivalent to the trivial cylinder, then $\widetilde{Z}^{Y}(M)-\varnothing$ starts in internal degree $i$. So, the LMO map induces a multiplicative map LMO : $\mathcal{C}_{g, 1} / Y_{i} \longrightarrow \mathcal{A}^{<} / \mathcal{A}_{\geq i}^{<}$for all $i \geq 1$ and, passing to the limit, we obtain

$$
\mathrm{LMO}: \widehat{\mathcal{C}}_{g, 1} \longrightarrow \mathcal{A}^{<} \text {. }
$$

Since $\widetilde{Z}^{Y}$ takes group-like values and since $\varphi$ and $s$ are Hopf algebra isomorphisms, the map (2.3) takes group-like values and so, by continuity of the coproduct, the map (2.4) does too. Thus, we get a Hopf algebra homomorphism

$$
\text { LMO : } \mathbb{Q}\left[\widehat{\mathcal{C}}_{g, 1}\right] \longrightarrow \mathcal{A}^{<} \text {. }
$$

There are two filtrations on the group algebra $\mathbb{Q}\left[\widehat{\mathcal{C}}_{g, 1}\right]$. On one hand, let $F$ be the filtration defined in the appendix at (A.1) and induced by the $\widehat{Y}$-filtration on the group $\widehat{\mathcal{C}}_{g, 1}$. On the other hand, let $F^{\prime}$ be the filtration defined by rational finite-type invariants: An $x \in \mathbb{Q}\left[\widehat{\mathcal{C}}_{g, 1}\right]$ is declared to belong to $F_{i}^{\prime} \mathbb{Q}\left[\widehat{\mathcal{C}}_{g, 1}\right]$ if $f(x)=0$ for any finite-type invariant $f: \mathcal{C}_{g, 1} \rightarrow \mathbb{Q}$ of degree at most $i-1$. By clasper calculus, it can be proved that $F=F^{\prime}$ (see [13, 24]). Since $\widetilde{Z}^{Y}$ is universal among rational finite-type invariants [4], (2.5) induces a monomorphism

$$
\text { LMO : } \mathbb{Q}\left[\widehat{\mathcal{C}}_{g, 1}\right] / F_{i} \mathbb{Q}\left[\widehat{\mathcal{C}}_{g, 1}\right] \longrightarrow \mathcal{A}^{<} / \mathcal{A}_{\geq i}^{<} .
$$

Actually, this map is an isomorphism since it is bijective at the graded level [4]. Thus, passing to the limit, we finally obtain an isomorphism

$$
\text { LMO : } \widehat{\mathbb{Q}}\left[\widehat{\mathcal{C}}_{g, 1}\right] \longrightarrow \mathcal{A}^{<}
$$

of complete Hopf algebras, where $\widehat{\mathbb{Q}}\left[\widehat{\mathcal{C}}_{g, 1}\right]$ denotes the completion of $\mathbb{Q}\left[\widehat{\mathcal{C}}_{g, 1}\right]$ with respect to the filtration $F$. Thus, we deduce the following

Theorem 2.3. Let $\mathcal{G}\left(\widehat{\mathcal{C}}_{g, 1}\right)$ be the Malcev completion of the group $\widehat{\mathcal{C}}_{g, 1}$ endowed with the $\widehat{Y}$-filtration, and let $\mathcal{P}\left(\widehat{\mathcal{C}}_{g, 1}\right)$ be its Malcev Lie algebra. Then, the LMO invariant induces an isomorphism of filtered groups

$$
\mathrm{LMO}: \mathcal{G}\left(\widehat{\mathcal{C}}_{g, 1}\right) \stackrel{\simeq}{\longrightarrow} \mathrm{G}\left(\mathcal{A}^{<}\right)
$$

as well as an isomorphism of filtered Lie algebras

$$
\mathrm{LMO}: \mathcal{P}\left(\widehat{\mathcal{C}}_{g, 1}\right) \stackrel{\simeq}{\longrightarrow} \mathrm{P}\left(\mathcal{A}^{<}\right) .
$$

The second part of this statement and Theorem A.8 gives back Theorem 1.2. Besides, it proves that the filtration on the Malcev Lie algebra of $\widehat{\mathcal{C}}_{g, 1}$ comes from a grading, which is not true for an arbitrary filtered group. 


\section{The Lie Algebra of SYMPleCtid JaCOBi DiAgRAmS}

In this section, we define the algebra of symplectic Jacobi diagrams, which is isomorphic to the algebras $\mathcal{A}^{<}\left(H_{\mathbb{Q}}\right)$ and is more convenient in some occasions. We interpret the multiplication of symplectic Jacobi diagrams as an analogue of the Moyal-Weyl product.

3.1. The algebra of symplectic Jacobi diagrams. We define the following vector space

$$
\mathcal{A}\left(H_{\mathbb{Q}}\right):=\frac{\mathbb{Q} \cdot\left\{\begin{array}{c}
\text { Jacobi diagrams without strut component } \\
\text { and with external vertices colored by } H_{\mathbb{Q}}
\end{array}\right\}}{\mathrm{AS}, \text { IHX, multilinearity }},
$$

which is also simply denoted by $\mathcal{A}$. It is graded by the internal degree of Jacobi diagrams, and its degree completion is also denoted by $\mathcal{A}\left(H_{\mathbb{Q}}\right)$.

There is a graded linear map

$$
\chi: \mathcal{A} \longrightarrow \mathcal{A}^{<}
$$

defined, for all Jacobi diagram $D \in \mathcal{A}$ with $e$ external vertices, by

$$
\chi(D):=\frac{1}{e !} \cdot(\text { sum of all ways of ordering the } e \text { external vertices of } D) .
$$

Proposition 3.1. The "symmetrization" map $\chi$ is an isomorphism. Its inverse is given on a Jacobi diagram $D \in \mathcal{A}^{<}$, with external vertices $v_{1}<\cdots<v_{e}$ colored by $c\left(v_{1}\right), \ldots, c\left(v_{e}\right) \in H_{\mathbb{Q}}$ respectively, by the formula

$$
\chi^{-1}(D)=\sum_{p=0}^{[e / 2]} \frac{1}{2^{p}} \sum_{\left\{i_{1}, j_{1}\right\}, \ldots,\left\{i_{p}, j_{p}\right\}} \prod_{k=1}^{p} \omega\left(c\left(v_{i_{k}}\right), c\left(v_{j_{k}}\right)\right) \cdot D_{\left(v_{i_{1}}=v_{j_{1}}, \ldots, v_{i_{p}}=v_{j_{p}}\right)} .
$$

Here, the second sum is taken over all ways of doing p pairings $\left\{i_{1}, j_{1}\right\}, \ldots,\left\{i_{p}, j_{p}\right\}$ inside the set $\{1, \ldots, e\}$ (with $i_{1}<\cdots<i_{p}$ and $i_{1}<j_{1}, \ldots, i_{p}<j_{p}$ ), and the diagram $D_{\left(v_{i_{1}}=v_{j_{1}}, \ldots, v_{i_{p}}=v_{j_{p}}\right)}$ is obtained from $D$ by gluing the vertices that are paired and by forgetting the order.

Proof. Let $\sigma(D)$ be the quantity defined by the right term of (3.1), for all Jacobi diagram $D$ colored by $H_{\mathbb{Q}}$ and with external vertices $v_{1}<\cdots<v_{e}$. Let $(l, l+1) \cdot D$ be the same diagram, but with the order of $v_{l}$ and $v_{l+1}$ reversed. Then, in the difference $\sigma(D)-\sigma((l, l+1) \cdot D)$, all terms cancel except for those corresponding to pairings that match $v_{l}$ and $v_{l+1}$ :

$$
\begin{aligned}
& \sigma(D)-\sigma((l, l+1) \cdot D) \\
& =\sum_{p=0}^{[e / 2]} \frac{1}{2^{p-1}} \sum_{\substack{\left\{i_{1}, j_{1}\right\}, \ldots,\left\{i_{p}, j_{p}\right\} \\
\exists r,\left(i_{r}, j_{r}\right)=(l, l+1)}} \prod_{k=1}^{p} \omega\left(c\left(v_{i_{k}}\right), c\left(v_{j_{k}}\right)\right) \cdot D_{\left(v_{i_{1}}=v_{j_{1}}, \ldots, v_{i_{p}}=v_{j_{p}}\right)} \\
& =\omega\left(c\left(v_{l}\right), c\left(v_{l+1}\right)\right) \cdot \sigma\left(D_{\left(v_{l}=v_{l+1}\right)}\right) .
\end{aligned}
$$

Thus, the STU-like relation is satisfied, and we get a linear map $\sigma: \mathcal{A}^{<} \rightarrow \mathcal{A}$.

Let $D$ be a Jacobi diagram colored by $H_{\mathbb{Q}}$ and with external vertices $v_{1}<\cdots<v_{e}$. The STU-like relation implies that $\chi(D)=D$ modulo some terms with fewer external vertices. (Here, the $D$ to which $\chi$ applies is obtained from $D$ by forgetting the order.) Moreover, $\chi(D)=D$ if $D$ has no external vertex $(e=0)$. This proves, by an induction on $e$, that $D$ belongs to the image of $\chi$. So, $\chi$ is surjective. 
Thus, it is enough to prove that $\sigma \circ \chi$ is the identity. For this, we define the space

$$
\widetilde{\mathcal{A}}\left(H_{\mathbb{Q}}\right):=\frac{\mathbb{Q} \cdot\left\{\begin{array}{l}
\text { Jacobi diagrams without strut component and with } \\
\text { external vertices totally ordered and colored by } H_{\mathbb{Q}}
\end{array}\right\}}{\mathrm{AS}, \text { IHX, multilinearity }},
$$

which we simply denote by $\widetilde{\mathcal{A}}$. (The space $\widetilde{\mathcal{A}}\left(H_{\mathbb{Q}}\right)$ is used also in the proof of Theorem 3.8.) Thus, the quotient of $\widetilde{\mathcal{A}}$ by the STU-like relation is $\mathcal{A}^{<}$while its quotient by the "forgetting orders" relation is $\mathcal{A}$. Let $\widetilde{\gamma}: \widetilde{\mathcal{A}} \rightarrow \widetilde{\mathcal{A}}$ be the linear map defined on each Jacobi diagram $D$ with external vertices $v_{1}<\cdots<v_{e}$ by

$$
\widetilde{\gamma}(D):=\sum_{1 \leq i<j \leq e} \omega\left(c\left(v_{i}\right), c\left(v_{j}\right)\right) \cdot D_{\left(v_{i}=v_{j}\right)}
$$

if $e \geq 2$, and by $\widetilde{\gamma}(D)=0$ if $e=0,1$. Then, we define $\widetilde{\sigma}: \widetilde{\mathcal{A}} \rightarrow \widetilde{\mathcal{A}}$ by

$$
\widetilde{\sigma}:=\exp _{\circ}(\widetilde{\gamma} / 2)=\sum_{k \geq 0} \frac{\widetilde{\gamma}^{k}}{2^{k} k !}
$$

Let also $\widetilde{\chi}: \widetilde{\mathcal{A}} \rightarrow \widetilde{\mathcal{A}}$ be the "symmetrization" map sending all Jacobi diagram $D$ to

$$
\widetilde{\chi}(D)=\frac{1}{e !} \cdot(\text { sum of all ways of permuting the } e \text { external vertices of } D) \text {. }
$$

The following diagram is commutative:

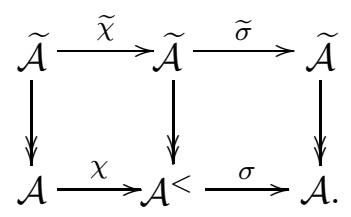

Since $\widetilde{\gamma} \circ \tilde{\chi}=0$, we have $\widetilde{\sigma} \circ \tilde{\chi}=\tilde{\chi}$, which implies that $\sigma \circ \chi=\mathrm{Id}$.

Thus, we can pull back by $\chi$ the product on $\mathcal{A}^{<}$to an associative multiplication $\star$ on the space $\mathcal{A}$, i.e. we set

$$
D \star E:=\chi^{-1}(\chi(D)\llcorner\chi(E))
$$

for all $D, E \in \mathcal{A}$. More generally, the full Hopf algebra structure on $\mathcal{A}^{<}$gives one for $\mathcal{A}$. The comultiplication in $\mathcal{A}$ is given on a Jacobi diagram $D$ by

$$
\Delta(D)=\sum_{D=D^{\prime} \sqcup D^{\prime \prime}} D^{\prime} \otimes D^{\prime \prime},
$$

the counit is given by $\varepsilon(D)=\delta_{D, \emptyset}$, and the antipode is the unique algebra antiautomorphism satisfying $S(D)=-D$ if $D$ is connected and non-empty. The primitive part $\mathrm{P}(\mathcal{A})$ of $\mathcal{A}$ is the subspace $\mathcal{A}^{c}$ spanned by the connected diagrams.

Definition 3.2. The Hopf algebra of symplectic Jacobi diagrams is $(\mathcal{A}, \varnothing, \star, \varepsilon, \Delta, S)$.

The multiplication $\star$ of Jacobi diagrams can also be defined directly as follows:

Proposition 3.3. Let $D, E \in \mathcal{A}$ be Jacobi diagrams colored by $H_{\mathbb{Q}}$, and whose sets of external vertices are denoted by $V$ and $W$ respectively. Then, we have

$$
D \star E=\sum_{\substack{V^{\prime} \subset V, W^{\prime} \subset W \\ \beta: V^{\prime} \stackrel{\simeq}{\longrightarrow} W^{\prime}}} \frac{1}{2^{\left|V^{\prime}\right|}} \cdot \prod_{v \in V^{\prime}} \omega(c(v), c(\beta(v))) \cdot\left(D \cup_{\beta} E\right) .
$$


Here, the sum is taken over all ways of identifying a subset $V^{\prime}$ of $V$ with a subset $W^{\prime}$ of $W$, and $D \cup_{\beta} E$ is obtained from $D \sqcup E$ by gluing each vertex $v \in V^{\prime}$ to $\beta(v) \in W^{\prime}$.

Consequently, the commutator $[D, E]_{\star}=D \star E-E \star D$ of $D$ and $E$ is given by

$$
[D, E]_{\star}=\sum_{\substack{\beta: V \supset V^{\prime} \simeq V^{\prime} \\\left|V^{\prime}\right|=\left|W^{\prime}\right| \equiv 1 \bmod 2}} \frac{1}{2^{\left|V^{\prime}\right|-1}} \prod_{v \in V^{\prime}} \omega(c(v), c(\beta(v))) \cdot\left(D \cup_{\beta} E\right),
$$

where the sum is taken over all ways of identifying a subset $V^{\prime}$ of $V$ of odd cardinality with a subset $W^{\prime}$ of $W$.

Proof of Proposition 3.3. Denote the external vertices of $D$ by $v_{1}, \ldots, v_{d}$, and those of $E$ by $w_{1}, \ldots, w_{e}$. By $(3.3)$, we have

$$
D \star E=\sum_{\delta \in S_{d}, \varepsilon \in S_{e}} \frac{1}{d ! \cdot e !} \cdot \chi^{-1}\left(D^{\delta}<E^{\varepsilon}\right),
$$

where $\delta$ is a permutation of $\{1, \ldots, d\}$ and $D^{\delta} \in \mathcal{A}^{<}$is obtained from $D$ by ordering its external vertices as $v_{\delta(1)}<\cdots<v_{\delta(d)}$. The diagram $E^{\varepsilon}$ is defined similarly from the permutation $\varepsilon$ of $\{1, \ldots, e\}$. If we apply formula (3.1) to $\chi^{-1}\left(D^{\delta} \leq E^{\varepsilon}\right)$, two kinds of terms appear in the resulting sum: Either, the gluings performed on $D^{\delta} \zeta E^{\varepsilon}$ are all "mixed", either at least one gluing is not mixed and involves, say, two external vertices of $D^{\delta}$. In the latter case, the corresponding term will appear with an opposite sign in $\chi^{-1}\left(D^{\tau \circ \delta}<E^{\varepsilon}\right)$ where $\tau \in S_{d}$ is the transposition of the indices of those two external vertices. Thus, we can assume that formula (3.1) applied to $\chi^{-1}\left(D^{\delta} \leq E^{\varepsilon}\right)$ involves only "mixed" gluing, in which case the ordering of the external vertices in $D^{\delta}$ and in $E^{\varepsilon}$ do not matter. The conclusion follows.

There is an obvious action of $\operatorname{Sp}\left(H_{\mathbb{Q}}\right)$ on $\mathcal{A}\left(H_{\mathbb{Q}}\right)$, such that $\chi$ is $\operatorname{Sp}\left(H_{\mathbb{Q}}\right)$-equivariant. Thus, the Hopf algebra structure on $\mathcal{A}\left(H_{\mathbb{Q}}\right)$ is compatible with this $\operatorname{Sp}\left(H_{\mathbb{Q}}\right)$-action. In particular, the Lie bracket $[-,-]_{\star}$ on $\mathcal{A}^{c}\left(H_{\mathbb{Q}}\right)$ is $\operatorname{Sp}\left(H_{\mathbb{Q}}\right)$-equivariant.

Remark 3.4. Garoufalidis and Levine [8] attempted to define a Lie bracket on the graded space $\mathcal{A}^{c}$, but, as pointed out by Habegger and Sorger [12], their Lie bracket is not $\operatorname{Sp}\left(H_{\mathbb{Q}}\right)$-equivariant so that [8, Theorem 6] fails. Yet the approach in [8] can be fixed at the tree level, as is done in $[12, \S 3]$.

3.2. Loop filtration. The loop degree of a Jacobi diagram is defined to be its first Betti number. ${ }^{3}$ For example, the loop degree of a tree diagram is 0 , and the loop degree of is 2. The loop degree is additive under disjoint union of diagrams.

Let $\mathcal{F}_{k}\left(\mathcal{A}^{<}\right)$be the subspace of $\mathcal{A}^{<}$spanned by the Jacobi diagrams of loop degree at least $k$. We have a filtration

$$
\mathcal{A}^{<}=\mathcal{F}_{0}\left(\mathcal{A}^{<}\right) \supset \mathcal{F}_{1}\left(\mathcal{A}^{<}\right) \supset \mathcal{F}_{2}\left(\mathcal{A}^{<}\right) \supset \cdots .
$$

This filtration induces a filtration on $\mathcal{A}_{i}^{<}$, for each $i \geq 1$, and is an algebra filtration:

$$
\mathcal{F}_{k}\left(\mathcal{A}^{<}\right) \leq \mathcal{F}_{l}\left(\mathcal{A}^{<}\right) \subset \mathcal{F}_{k+l}\left(\mathcal{A}^{<}\right) .
$$

Similarly, let $\mathcal{F}_{k}(\mathcal{A})$ be the subspace of $\mathcal{A}$ spanned by the Jacobi diagrams of loop degree at least $k$. Again, we have a filtration

$$
\mathcal{A}=\mathcal{F}_{0}(\mathcal{A}) \supset \mathcal{F}_{1}(\mathcal{A}) \supset \mathcal{F}_{2}(\mathcal{A}) \supset \cdots
$$

\footnotetext{
${ }^{3}$ In the literature, the loop degree is sometimes defined as the first Betti number minus 1.
} 
which induces a filtration on $\mathcal{A}_{i}$, for each $i \geq 1$, and is an algebra filtration:

$$
\mathcal{F}_{k}(\mathcal{A}) \star \mathcal{F}_{l}(\mathcal{A}) \subset \mathcal{F}_{k+l}(\mathcal{A}) .
$$

The above two filtrations are connected by the symmetrization isomorphism:

$$
\chi\left(\mathcal{F}_{k}(\mathcal{A})\right)=\mathcal{F}_{k}\left(\mathcal{A}^{<}\right) .
$$

The above algebra filtrations also induce Lie algebra filtrations on $\mathcal{A}^{<, c}$ and $\mathcal{A}^{c}$. To be more specific, if we define

$$
\begin{gathered}
\mathcal{F}_{k}\left(\mathcal{A}^{<, c}\right):=\mathcal{F}_{k}\left(\mathcal{A}^{<}\right) \cap \mathcal{A}^{<, c}, \\
\mathcal{F}_{k}\left(\mathcal{A}^{c}\right):=\mathcal{F}_{k}(\mathcal{A}) \cap \mathcal{A}^{c},
\end{gathered}
$$

then we have $\left[\mathcal{F}_{k}, \mathcal{F}_{l}\right] \subset \mathcal{F}_{k+l}$ in the two cases.

The "loop filtrations" defined above are closely related to clasper calculus. For example, one can prove that if a graph clasper $C$ in the trivial cylinder over $\Sigma_{g, 1}$ has $k$ loops, then the LMO invariant of the homology cylinder obtained by surgery along $C$ is contained in $\mathcal{F}_{k}\left(\mathcal{A}^{<}\right)$.

For each $i \geq 1, \mathcal{A}_{i}^{c}$ is graded as a vector space by the loop degree:

$$
\mathcal{A}_{i}^{c}=\bigoplus_{0 \leq k \leq d_{i}} \mathcal{A}_{i, k}^{c}
$$

where $d_{i}=(i+2) / 2$ if $i$ is even, and $d_{i}=(i-1) / 2$ if $i$ is odd. Set

$$
\mathcal{A}_{i, \mathrm{ev}}^{c}:=\bigoplus_{k \text { even }} \mathcal{A}_{i, k}^{c} \quad \text { and } \quad \mathcal{A}_{i, \mathrm{od}}^{c}:=\bigoplus_{k \text { odd }} \mathcal{A}_{i, k}^{c} .
$$

Then (3.4) implies that $\mathcal{A}_{* \text { ev }}^{c}$ is a Lie subalgebra of $\mathcal{A}^{c}$, and that the decomposition

$$
\mathcal{A}^{c}=\mathcal{A}_{*, \mathrm{ev}}^{c} \oplus \mathcal{A}_{*, \mathrm{od}}^{c}
$$

defines a $\mathbb{Z} / 2 \mathbb{Z}$-graded Lie algebra structure on $\mathcal{A}^{c}$. In particular, we have

$$
a\left(H_{\mathbb{Q}}\right) \subset \mathcal{A}_{*, \text { ev }}^{c}\left(H_{\mathbb{Q}}\right),
$$

where $a\left(H_{\mathbb{Q}}\right)$ is the Lie subalgebra of $\mathcal{A}^{c}\left(H_{\mathbb{Q}}\right)$ generated by the degree 1 part $\mathcal{A}_{1}^{c}$.

3.3. Weight systems and the Moyal-Weyl product. Let us recall the definition of the Moyal-Weyl product. For this, we consider a $\mathbb{Q}$-vector space $V$ together with a symplectic form $s: V \otimes V \rightarrow \mathbb{Q}$.

Definition 3.5. The Weyl algebra generated by $V$ is the quotient of the tensor algebra of $V$ by the relations " $u \otimes v-v \otimes u=s(u, v)$ ":

$$
\mathcal{W}(V):=T(V) /\langle u \otimes v-v \otimes u-s(u, v) \mid u, v \in V\rangle_{\text {ideal }} .
$$

The "symmetrization" map $\chi: S(V) \longrightarrow \mathcal{W}(V)$ is defined by

$$
\chi\left(v_{1} \cdots v_{n}\right):=\frac{1}{n !} \sum_{\sigma \in S_{n}}\left\{v_{\sigma(1)} \otimes \cdots \otimes v_{\sigma(n)}\right\} .
$$

By formally the same argument as in Proposition 3.1, it can be shown that $\chi$ is an isomorphism, which justifies the following

Definition 3.6. The associative multiplication on the vector space $S(V)$ corresponding to $\otimes$ on $\mathcal{W}(V)$ is denoted by $\star$ and is called the Moyal-Weyl product. 
Remark 3.7. Recall that a deformation quantization of a Poisson algebra $(A, \cdot,\{-,-\})$ is a $\mathbb{Q}[[h]]$-linear associative multiplication $\star_{h}$ on the space $A[[h]]$, such that $a \star_{h} b=$ $a \cdot b+O(h)$ and $a \star_{h} b-b \star_{h} a=\{a, b\} \cdot h+O\left(h^{2}\right)$, for all $a, b \in A$. See [3].

We are considering here the commutative algebra $(S(V), \cdot)$ with Poisson bracket defined by $\{u, v\}:=s(u, v)$ for all $u, v \in V$. The Moyal-Weyl product usually refers to its deformation quantization

$$
\star_{h}: S(V)[[h]] \otimes S(V)[[h]] \longrightarrow S(V)[[h]]
$$

defined, for all $A, B \in S(V)$, by

$$
A \star_{h} B:=\sum_{l=0}^{\infty} \frac{h^{l}}{2^{l} l !} \sum_{\substack{i_{1}, \ldots, i_{l} \in\{1, \ldots, d\} \\ j_{1}, \ldots, j_{l} \in\{1, \ldots, d\}}}\left(\prod_{k=1}^{l} s\left(x_{i_{k}}, x_{j_{k}}\right)\right) \frac{\partial^{l} A}{\partial x_{i_{1}} \cdots \partial x_{i_{l}}} \frac{\partial^{l} B}{\partial x_{j_{1}} \cdots \partial x_{j_{l}}} .
$$

Here, a basis $\left(x_{1}, \ldots, x_{d}\right)$ of $V$ has been chosen so that $S(V)$ is identified with the polynomial algebra $\mathbb{Q}\left[x_{1}, \ldots, x_{d}\right]$. Using an analogue of Proposition 3.3 for the product $\star$ on $S(V)$, it is easily checked that $\star$ coincides with $\star_{h}$ at $h=1$.

To connect the multiplication $\star$ of symplectic Jacobi diagrams to the Moyal-Weyl product, we consider a metrized Lie algebra $\mathfrak{g}$. Thus, $\mathfrak{g}$ is a finite-dimensional Lie algebra together with a symmetric bilinear form $\kappa: \mathfrak{g} \times \mathfrak{g} \rightarrow \mathbb{Q}$, which is $\mathfrak{g}$-invariant and non-degenerate.

It is well-known that such a data defines a linear map $\mathcal{A}(\varnothing) \rightarrow \mathbb{Q}[[t]]$, called the weight system associated to $\mathfrak{g}$ : This is the case of homology spheres considered in [21] or, equivalently, the case of homology cylinders of genus $g=0$. This construction extends to higher genus as follows. First, we equip $\mathfrak{g} \otimes H_{\mathbb{Q}}$ with the symplectic form $\kappa \otimes \omega$, where $\omega$ is the intersection pairing on $\Sigma_{g, 1}$.

Theorem 3.8. We can define non-trivial algebra homomorphisms

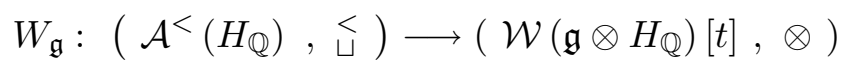

and

$$
W_{\mathfrak{g}}:\left(\mathcal{A}\left(H_{\mathbb{Q}}\right), \star\right) \longrightarrow\left(S\left(\mathfrak{g} \otimes H_{\mathbb{Q}}\right)[t], \star\right),
$$

sending the internal degree to the $t$-degree and such that the following diagram commutes in the category of graded algebras:

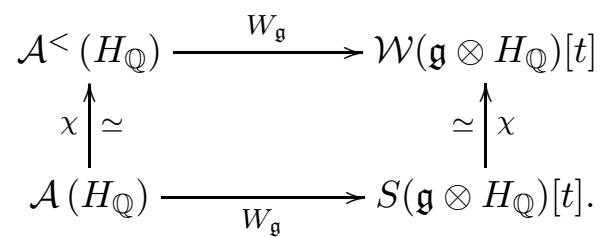

Proof. Let $K \in S^{2} \mathfrak{g}$ be the 2-tensor corresponding to $\kappa \in S^{2} \mathfrak{g}^{*} \simeq\left(S^{2} \mathfrak{g}\right)^{*}$ by the isomorphism $\mathfrak{g} \rightarrow \mathfrak{g}^{*}$ adjoint to $\kappa$. Let also $B \in \Lambda^{3} \mathfrak{g}^{*} \simeq\left(\Lambda^{3} \mathfrak{g}\right)^{*}$ be the alternating trilinear form defined by $x \wedge y \wedge z \mapsto \kappa([x, y], z)$.

Then, any Jacobi diagram $D$ whose external vertices are numbered from 1 to $e$ defines a tensor in $\mathfrak{g}^{\otimes e}$ : Each internal vertex is replaced by a copy of $B$, each edge by a copy of $K$ and contractions are performed. If $D$ is now colored by $H_{\mathbb{Q}}$, then we get a tensor $\widetilde{w}_{\mathfrak{g}}(D)$ in $\mathfrak{g}^{\otimes e} \otimes H_{\mathbb{Q}}^{\otimes e} \simeq\left(\mathfrak{g} \otimes H_{\mathbb{Q}}\right)^{\otimes e}$. Let $\widetilde{\mathcal{A}}\left(H_{\mathbb{Q}}\right)$ be the space of Jacobi diagrams defined at (3.2) and define a linear map

$$
\widetilde{W}_{\mathfrak{g}}: \widetilde{\mathcal{A}}\left(H_{\mathbb{Q}}\right) \longrightarrow T\left(\mathfrak{g} \otimes H_{\mathbb{Q}}\right)[t]
$$


by $D \mapsto \widetilde{w}_{\mathfrak{g}}(D) \cdot t^{i}$ for all Jacobi diagram $D$ with $i$ internal vertices. This map is welldefined since the antisymmetry and the Jacobi identity satisfied by the Lie bracket of $\mathfrak{g}$ are mapped to the AS and IHX relations, respectively, as usual. Observe that the ordered disjoint union $\leq$ defines an associative multiplication on $\widetilde{\mathcal{A}}\left(H_{\mathbb{Q}}\right)$, and that $\widetilde{W}_{\mathfrak{g}}$ is then multiplicative.

Because the contraction of $K \otimes \kappa \otimes K$ gives $K$, the map $\widetilde{W}_{\mathfrak{g}}$ induces a linear map $W_{\mathfrak{g}}: \mathcal{A}^{<}\left(H_{\mathbb{Q}}\right) \rightarrow \mathcal{W}\left(\mathfrak{g} \otimes H_{\mathbb{Q}}\right)[t]$. Obviously, the multiplicativity of the former implies the multiplicativity of the latter.

Clearly, the map $\widetilde{W}_{\mathfrak{g}}$ induces a linear map $W_{\mathfrak{g}}: \mathcal{A}\left(H_{\mathbb{Q}}\right) \rightarrow \mathrm{S}\left(\mathfrak{g} \otimes H_{\mathbb{Q}}\right)[t]$ as well. The commutativity of (3.7) follows from the definitions and shows that the multiplicativity of its top map implies that of its bottom map.

Remark 3.9. Since the form $\kappa$ is $\mathfrak{g}$-invariant, the above two maps $W_{\mathfrak{g}}$ actually take values on the $\mathfrak{g}$-invariant subspaces.

\section{Algebraic Description of the mapping Cylinder Construction}

In this section, we prove Theorem 1.4, which gives an algebraic description of the mapping cylinder construction $\mathbf{c}: \mathcal{I}_{g, 1} \rightarrow \mathcal{C}_{g, 1}$ at the level of graded Lie algebras. To start with, we shall describe the action of $\operatorname{Sp}\left(H_{\mathbb{Q}}\right)$ on the four graded Lie algebras of diagram (1.7).

4.1. Symplectic actions. We start by recalling how $\operatorname{Sp}\left(H_{\mathbb{Q}}\right)$ acts on $\operatorname{Gr}^{\Gamma} \mathcal{I}_{g, 1} \otimes \mathbb{Q}$. The conjugation action of the mapping class group $\mathcal{M}_{g, 1}$ on $\mathcal{I}_{g, 1}$ induces an $\operatorname{Sp}(H)$-action on $\operatorname{Gr}^{\Gamma} \mathcal{I}_{g, 1}$ such that the Lie bracket is $\operatorname{Sp}(H)$-equivariant. There is also the standard action of $\operatorname{Sp}(H)$ on $\Lambda^{3} H$, and the first Johnson homomorphism is $\operatorname{Sp}(H)$-equivariant: Thus, for $g \geq 3$, the Lie algebra map

$$
J: \operatorname{Lie}\left(\Lambda^{3} H_{\mathbb{Q}}\right) \longrightarrow \operatorname{Gr}^{\Gamma} \mathcal{I}_{g, 1} \otimes \mathbb{Q}
$$

is $\operatorname{Sp}(H)$-equivariant. It follows that $\operatorname{Ker}(J)$ is an $\operatorname{Sp}(H)$-submodule and so, by the "algebraicity lemma" of $[1, \S 2.2 .8]$, it is an $\operatorname{Sp}\left(H_{\mathbb{Q}}\right)$-submodule as well. Consequently, the Lie algebra epimorphism $J$ transports the action of $\operatorname{Sp}\left(H_{\mathbb{Q}}\right)$ on $\operatorname{Lie}\left(\Lambda^{3} H_{\mathbb{Q}}\right)$ onto an action of $\operatorname{Sp}\left(H_{\mathbb{Q}}\right)$ on $\operatorname{Gr}^{\Gamma} \mathcal{I}_{g, 1} \otimes \mathbb{Q}$, and this extends the canonical action of $\operatorname{Sp}(H)$.

Let us now precise how $\operatorname{Sp}\left(H_{\mathbb{Q}}\right)$ acts on the Lie algebra of homology cylinders.

Lemma 4.1. Assume that $g \geq 0$. There is a natural action of $\operatorname{Sp}(H)$ on the Lie algebra $\operatorname{Gr}^{Y} \mathcal{C}_{g, 1}$, which is compatible with the usual action of $\mathrm{Sp}(H)$ on $\mathrm{Gr}^{\Gamma} \mathcal{I}_{g, 1}$. Moreover, the Lie bracket of $\operatorname{Gr}^{Y} \mathcal{C}_{g, 1}$ is $\operatorname{Sp}(H)$-equivariant.

Proof. Let $\operatorname{Cob}\left(\Sigma_{g, 1}, \Sigma_{g, 1}\right)$ be the monoid of cobordisms from $\Sigma_{g, 1}$ to $\Sigma_{g, 1}$. The mapping cylinder construction defines an inclusion $\mathcal{M}_{g, 1} \hookrightarrow \operatorname{Cob}\left(\Sigma_{g, 1}, \Sigma_{g, 1}\right)$. Thus, $\mathcal{M}_{g, 1}$ acts on $\operatorname{Cob}\left(\Sigma_{g, 1}, \Sigma_{g, 1}\right)$ by conjugation:

$$
\mathcal{M}_{g, 1} \times \mathcal{C} o b\left(\Sigma_{g, 1}, \Sigma_{g, 1}\right) \ni(f, M) \longmapsto f \circ M \circ f^{-1} \in \mathcal{C} o b\left(\Sigma_{g, 1}, \Sigma_{g, 1}\right) .
$$

The Mayer-Vietoris theorem shows that this action preserves the submonoid $\mathcal{C}_{g, 1}$ of $\operatorname{Cob}\left(\Sigma_{g, 1}, \Sigma_{g, 1}\right)$. The $Y_{i}$-equivalence being generated by surgeries along graph claspers with $i$ nodes, this action also preserves the submonoid $Y_{i} \mathcal{C}_{g, 1}$ of $\mathcal{C}_{g, 1}$. This follows from the general fact

$$
\begin{aligned}
& \forall \text { graph clasper } G \subset \Sigma_{g, 1} \times[-1,1], \forall f \in \mathcal{M}_{g, 1}, \\
& \left(f \circ\left(\Sigma_{g, 1} \times[-1,1]\right) \circ f^{-1}, G\right) \cong\left(\Sigma_{g, 1} \times[-1,1],\left(f \times \operatorname{Id}_{[-1,1]}\right)(G)\right)
\end{aligned}
$$


about pairs (3-manifold with boundary, graph clasper). Therefore, the group $\mathcal{M}_{g, 1}$ acts on $\operatorname{Gr}^{Y} \mathcal{C}_{g, 1}$. But, inclusion (1.2) also shows that

$$
\forall f \in \mathcal{I}_{g, 1}, \forall M \in Y_{i} \mathcal{C}_{g, 1}, f \circ M \circ f^{-1} \sim_{Y_{i+1}} M .
$$

So, the action of $\mathcal{M}_{g, 1}$ on $\operatorname{Gr}^{Y} \mathcal{C}_{g, 1}$ factorizes to $\mathcal{M}_{g, 1} / \mathcal{I}_{g, 1} \simeq \operatorname{Sp}(H)$. The second statement of the lemma is easily checked.

Next, we have the following result generalizing the $\operatorname{Sp}(H)$-equivariance of Johnson's homomorphism:

Lemma 4.2. Let $g \geq 0$. The Lie algebra isomorphisms $\psi$ and LMO, defined in $\S 2.2$ and $\S 2.3$ respectively, are both $\mathrm{Sp}(H)$-equivariant.

So, we can transport the action of $\operatorname{Sp}\left(H_{\mathbb{Q}}\right)$ on $\mathcal{A}^{<, c}\left(H_{\mathbb{Q}}\right)$ to an action on $\mathrm{Gr}^{Y} \mathcal{C}_{g, 1} \otimes \mathbb{Q}$, which extends the natural action of $\operatorname{Sp}(H)$ given by Lemma 4.1 .

Proof of Lemma 4.2. It is enough to show that $\psi$ is $\operatorname{Sp}(H)$-equivariant. Let $D \in \mathcal{A}^{<, c}$ be a connected Jacobi diagram and let $F \in \operatorname{Sp}(H)$. Choose $f \in \mathcal{M}_{g, 1}$ which induces $F$ in homology. Then, we have

$$
\begin{aligned}
\psi(F \cdot D)= & \left\{\left(\Sigma_{g, 1} \times[-1,1]\right)_{C(F \cdot D)}\right\}=\left\{\left(\Sigma_{g, 1} \times[-1,1]\right)_{\left(f \times \operatorname{Id}_{[-1,1]}\right)(C(D))}\right\} \\
& \stackrel{(4.1)}{=}\left\{f \circ\left(\Sigma_{g, 1} \times[-1,1]\right)_{C(D)} \circ f^{-1}\right\}=F \cdot\{\psi(D)\} .
\end{aligned}
$$

Remark 4.3. The construction of the LMO functor in [4] and, a fortiori, the definition of the LMO homomorphism (2.1)

$$
\mathrm{LMO}=s \circ \varphi \circ \widetilde{Z}^{Y}: \mathcal{C}_{g, 1} \longrightarrow \mathcal{A}^{<}
$$

depends on a choice of meridian and parallel curves $\left(\alpha_{1}, \ldots, \alpha_{g}, \beta_{1}, \ldots, \beta_{g}\right)$ shown in Figure 4.1. Another choice $\left(\alpha_{1}^{\prime}, \ldots, \alpha_{g}^{\prime}, \beta_{1}^{\prime}, \ldots, \beta_{g}^{\prime}\right)$ would lead to "another" invariant

$$
\mathrm{LMO}^{\prime}: \mathcal{C}_{g, 1} \longrightarrow \mathcal{A}^{<} \text {. }
$$

Let $f: \Sigma_{g, 1} \rightarrow \Sigma_{g, 1}$ be a homeomorphism sending the curves $\alpha, \beta$ to $\alpha^{\prime}, \beta^{\prime}$ respectively.

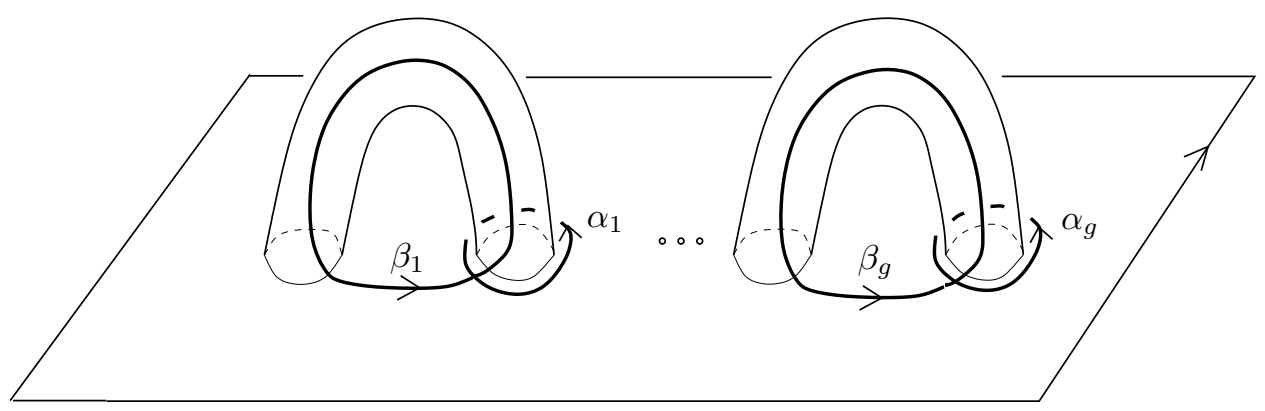

Figure 4.1. The surface $\Sigma_{g, 1}$ and its system of meridians and parallels $(\alpha, \beta)$.

Then, the connection between the latter invariant and the former one is as follows:

$$
\forall M \in \mathcal{C}_{g, 1}, \operatorname{LMO}^{\prime}(M)=f_{*} \cdot \operatorname{LMO}\left(f^{-1} \cdot M \cdot f\right),
$$

where $f_{*} \in \operatorname{Sp}(H)$ denotes the action of $f$ on $H$. Therefore, Lemma 4.2 says that the LMO homomorphism (2.1) does not depend on the choice of $(\alpha, \beta)$ at the graded level. 
4.2. The mapping cylinder construction at the level of graded Lie algebras. We can prove Theorem 1.4. Let $Y: \operatorname{Lie}\left(\Lambda^{3} H_{\mathbb{Q}}\right) \rightarrow \mathcal{A}^{<, c}\left(H_{\mathbb{Q}}\right)$ be the Lie algebra homomorphism defined by identifying $\Lambda^{3} H_{\mathbb{Q}}$ to $\mathcal{A}_{1}^{<, c}\left(H_{\mathbb{Q}}\right)$ as follows:

$$
a \wedge b \wedge c \mapsto a b c
$$

the total ordering of the external vertices being irrelevant in this case. Since the Lie bracket of $\mathcal{A}^{<, c}$ is equivariant under the action of $\operatorname{Sp}\left(H_{\mathbb{Q}}\right)$, the map $Y$ is equivariant as well. So, we have the following diagram in the category of graded Lie algebras with $\operatorname{Sp}\left(H_{\mathbb{Q}}\right)$-actions:

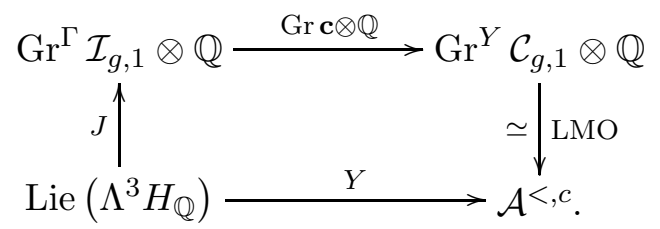

This commutes in degree 1 since the $Y$-part of the LMO invariant $\operatorname{Gr} \widetilde{Z}^{Y}$ defined in [4] corresponds to the first Johnson homomorphism. Since the Lie algebra Lie $\left(\Lambda^{3} H_{\mathbb{Q}}\right)$ is generated by its degree 1 elements, that diagram commutes in any degree. This completes the proof of Theorem 1.4.

\section{The Degree two CASE}

In this section, we recall a few facts about classical representation theory of $\mathrm{Sp}_{2 g} \mathbb{C}$. We also recall the quadratic relations of the Torelli Lie algebra, as given explicitly in [12]. Then, we compute the Lie bracket of $\mathcal{A}^{c}$ in degree $1+1$, which allows us to prove Theorem 1.5.

5.1. Representation theory of $\mathrm{Sp}_{2 g} \mathbb{C}$. For basics of the representation theory of the Lie group $\mathrm{Sp}_{2 g} \mathbb{C}$, the reader is referred to $[6, \S \S 16-17]$, the notations of which we will follow. Thus, we denote

$$
\mathrm{Sp}_{2 g} \mathbb{A}:=\left\{M \in \mathrm{GL}_{2 g} \mathbb{A}:{ }^{t} M \Omega M=\Omega\right\}, \quad \text { where } \Omega:=\left(\begin{array}{cc}
0 & I_{g} \\
-I_{g} & 0
\end{array}\right) \text { and } \mathbb{A}:=\mathbb{Z}, \mathbb{Q}, \mathbb{C} \text {. }
$$

The representation theory of the complex Lie group $\mathrm{Sp}_{2 g} \mathbb{C}$ is the same as that of its Lie algebra

$$
\mathfrak{s p}_{2 g} \mathbb{C}=\left\{X \in \mathfrak{g l}_{2 g} \mathbb{C}:{ }^{t} X \Omega+\Omega X=0\right\} .
$$

The diagonal matrices in $\mathfrak{s p}_{2 g} \mathbb{C}$ form a Cartan subalgebra $\mathfrak{h}$. Set $H_{i}:=E_{i, i}-E_{g+i, g+i}$, where $E_{i, j}$ denotes the elementary matrix with only one 1 in position $(i, j)$. Then, $\left(H_{1}, \ldots, H_{g}\right)$ is a basis of $\mathfrak{h}$ whose dual basis of $\mathfrak{h}^{*}$ is denoted by $\left(L_{1}, \ldots, L_{g}\right)$.

With respect to the above Cartan subalgebra $\mathfrak{h}$, the set of roots of $\mathfrak{s p}_{2 g} \mathbb{C}$ is $R=$ $\left\{ \pm L_{i} \pm L_{j}\right\}$, and here are the corresponding eigenvectors:

\begin{tabular}{c|c} 
eigenvalue & eigenvector \\
\hline$L_{i}-L_{j}(i \neq j)$ & $X_{i, j}:=E_{i, j}-E_{g+j, g+i}$ \\
\hline$L_{i}+L_{j}(i \neq j)$ & $Y_{i, j}:=E_{i, g+j}+E_{j, g+i}$ \\
\hline$-L_{i}-L_{j}(i \neq j)$ & $Z_{i, j}:=E_{g+i, j}+E_{g+j, i}$ \\
\hline $2 L_{i}$ & $U_{i}:=E_{i, g+i}$ \\
\hline$-2 L_{i}$ & $V_{i}:=E_{g+i, i}$
\end{tabular}


One can declare the positive roots to be $R^{+}=\left\{L_{i}+L_{j} \mid i \leq j\right\} \cup\left\{L_{i}-L_{j} \mid i<j\right\}$, so that the primitive positive roots are the $L_{i}-L_{i+1}$ 's, for $i=1, \ldots, g-1$, and $2 L_{g}$.

The weight lattice of $\mathfrak{s p}_{2 g} \mathbb{C}$ is spanned by the $L_{i}$ 's, and the fundamental weights are

$$
\omega_{1}:=L_{1}, \omega_{2}:=L_{1}+L_{2}, \ldots, \omega_{g}:=L_{1}+L_{2}+\cdots+L_{g} .
$$

Then, to each $g$-tuple of non-negative integers $\left(a_{1}, \ldots, a_{g}\right)$ corresponds a unique irreducible representation $\Gamma_{a_{1} \omega_{1}+\cdots+a_{g} \omega_{g}}$ of $\mathfrak{s p}_{2 g} \mathbb{C}$ with highest weight $a_{1} \omega_{1}+\cdots+a_{g} \omega_{g}$. The data $\left(a_{1}, \ldots, a_{g}\right)$ can be thought of as the Young diagram with $a_{i}$ columns of height $i$ or, equivalently, as the partition $\lambda=\left(a_{g}+\cdots+a_{2}+a_{1}, a_{g}+\cdots+a_{2}, \ldots, a_{g}\right)$ of length $|\lambda|=\sum_{i=1}^{g} i \cdot a_{i}$. Thus, irreducible $\mathfrak{s p}_{2 g} \mathbb{C}$-modules or, equivalently, irreducible $\mathrm{Sp}_{2 g} \mathbb{C}$-modules are indexed by partitions $\lambda$ with no more than $g$ parts.

Actually, the $\mathfrak{s p}_{2 g} \mathbb{C}$-module $\Gamma_{a_{1} \omega_{1}+\cdots+a_{g} \omega_{g}}$ can be realized as the "symplectic" Schur module $\mathbb{S}_{\langle\lambda\rangle} \mathbb{C}^{2 g}$ associated to $\lambda$, i.e. the intersection in $\left(\mathbb{C}^{2 g}\right)^{\otimes|\lambda|}$ of the ordinary Schur module $\mathbb{S}_{\lambda} \mathbb{C}^{2 g}$ with the kernels of all possible contractions $\left(\mathbb{C}^{2 g}\right)^{\otimes|\lambda|} \rightarrow\left(\mathbb{C}^{2 g}\right)^{\otimes(|\lambda|-2)}$ defined by the symplectic form $\Omega$. It follows that each representation $\Gamma_{a_{1} \omega_{1}+\cdots+a_{g} \omega_{g}}$ exists with rational coefficients, and defines an irreducible $\mathrm{Sp}_{2 g} \mathbb{Q}$-module as well.

Example 5.1. For all $k=1, \ldots, g$, the fundamental representation $\Gamma_{\omega_{k}}$ is the symplectic Schur module given by the Young diagram with only one column of height $k$. So, $\Gamma_{\omega_{k}}$ is the kernel of the contraction map

$$
\Lambda^{k} \mathbb{C}^{2 g} \longrightarrow \Lambda^{k-2} \mathbb{C}^{2 g}, v_{1} \wedge \cdots \wedge v_{k} \longmapsto \sum_{i<j}(-1)^{i+j} \Omega\left(v_{i}, v_{j}\right) \cdot v_{1} \wedge \cdots \widehat{v}_{i} \cdots \widehat{v}_{j} \cdots \wedge v_{k}
$$

In the sequel, we will meet some $\mathrm{Sp}_{2 g} \mathbb{C}$-modules that are restrictions of $\mathrm{GL}_{2 g} \mathbb{C}$ modules via the canonical inclusion $\mathrm{Sp}_{2 g} \mathbb{C} \subset \mathrm{GL}_{2 g} \mathbb{C}$. In particular, the ordinary Schur module $\mathbb{S}_{\lambda} \mathbb{C}^{2 g}$ can be regarded as an $\mathrm{Sp}_{2 g} \mathbb{C}$-module, and a "restriction formula" by Littlewood gives its irreducible decomposition when the partition $\lambda$ has no more than $g$ parts:

$$
\mathbb{S}_{\lambda} \mathbb{C}^{2 g} \simeq \bigoplus_{\mu} N_{\mu \lambda} \cdot \mathbb{S}_{\langle\mu\rangle} \mathbb{C}^{2 g}
$$

Here, the sum is over all partitions $\mu$ with no more than $g$ parts and

$$
N_{\mu \lambda}=\sum_{\eta} N_{\eta \mu \lambda}
$$

is the sum of the Littlewood-Richardson coefficients $N_{\eta \mu \lambda}$ over all partitions $\eta$ with each part occurring an even number of times. See $[23,(4.4)]$ or $[6,(25.39)]$ for details.

For example, the irreducible decomposition of $\Lambda^{2} \Lambda^{3} \mathbb{C}^{2 g}$ as an $\mathrm{Sp}_{2 g} \mathbb{C}$-module can be computed using this method. We restrict to the case $g \geq 3$ since this will be enough for our purposes.

Lemma 5.2. We have the following isomorphism of $\mathrm{Sp}_{2 g} \mathbb{C}$-modules:

$$
\Lambda^{2} \Lambda^{3} \mathbb{C}^{2 g} \simeq \begin{cases}2 \Gamma_{0}+3 \Gamma_{\omega_{2}}+\Gamma_{2 \omega_{2}}+\Gamma_{\omega_{1}+\omega_{3}}+2 \Gamma_{\omega_{4}}+\Gamma_{\omega_{2}+\omega_{4}}+\Gamma_{\omega_{6}} & \text { if } g \geq 6 \\ 2 \Gamma_{0}+3 \Gamma_{\omega_{2}}+\Gamma_{2 \omega_{2}}+\Gamma_{\omega_{1}+\omega_{3}}+2 \Gamma_{\omega_{4}}+\Gamma_{\omega_{2}+\omega_{4}} & \text { if } g=5 \\ 2 \Gamma_{0}+3 \Gamma_{\omega_{2}}+\Gamma_{2 \omega_{2}}+\Gamma_{\omega_{1}+\omega_{3}}+\Gamma_{\omega_{4}}+\Gamma_{\omega_{2}+\omega_{4}} & \text { if } g=4 \\ 2 \Gamma_{0}+2 \Gamma_{\omega_{2}}+\Gamma_{2 \omega_{2}}+\Gamma_{\omega_{1}+\omega_{3}} & \text { if } g=3\end{cases}
$$

Proof. The irreducible decomposition of $\Lambda^{2} \Lambda^{3} \mathbb{C}^{2 g}$ as a $\mathrm{GL}_{2 g} \mathbb{C}$-module can be deduced from Pieri's formula for all $g \geq 0$ :

$$
\Lambda^{2} \Lambda^{3} \mathbb{C}^{2 g} \simeq \mathbb{S}_{(1,1,1,1,1,1)} \mathbb{C}^{2 g} \oplus \mathbb{S}_{(2,2,1,1)} \mathbb{C}^{2 g},
$$


see [6, Exercice 15.32]. We deduce from Example 5.1 that

$$
\mathbb{S}_{(1,1,1,1,1,1)} \mathbb{C}^{2 g}=\Lambda^{6} \mathbb{C}^{2 g} \simeq\left\{\begin{aligned}
& \Gamma_{0}+\Gamma_{\omega_{2}}+\Gamma_{\omega_{4}}+\Gamma_{\omega_{6}} & \text { if } g \geq 6, \\
\Lambda^{4} \mathbb{C}^{2 g} & \simeq \Gamma_{0}+\Gamma_{\omega_{2}}+\Gamma_{\omega_{4}} & \text { if } g=5, \\
\Lambda^{2} \mathbb{C}^{2 g} & \simeq \Gamma_{0}+\Gamma_{\omega_{2}} & \text { if } g=4, \\
\mathbb{C} & \simeq \Gamma_{0} & \text { if } g=3 .
\end{aligned}\right.
$$

Moreover, Littlewood's restriction formula (5.1) shows $^{4}$ that

$$
\mathbb{S}_{(2,2,1,1)} \mathbb{C}^{2 g} \simeq \begin{cases}\Gamma_{0}+2 \Gamma_{\omega_{2}}+\Gamma_{2 \omega_{2}}+\Gamma_{\omega_{1}+\omega_{3}}+\Gamma_{\omega_{4}}+\Gamma_{\omega_{2}+\omega_{4}} & \text { if } g \geq 4 \\ \Gamma_{0}+2 \Gamma_{\omega_{2}}+\Gamma_{2 \omega_{2}}+\Gamma_{\omega_{1}+\omega_{3}} & \text { if } g=3\end{cases}
$$

The conclusion follows.

5.2. Quadratic relations of the Torelli Lie algebra. Let $\left(\alpha_{1}, \ldots, \alpha_{g}, \beta_{1}, \ldots, \beta_{g}\right)$ be a system of meridians and parallels on the surface $\Sigma_{g, 1}$, as shown in Figure 4.1. This defines a symplectic basis of $H_{\mathbb{Q}}$, so that $\operatorname{Sp}\left(H_{\mathbb{Q}}\right)$ is identified with $\mathrm{Sp}_{2 g} \mathbb{Q}$.

By abuse of notation, let $\omega \in \Lambda^{2} H_{\mathbb{Q}}$ denote the bivector dual to the symplectic form $\omega$, namely

$$
\omega:=\sum_{i=1}^{g} \alpha_{i} \wedge \beta_{i}
$$

Define $r_{1}, r_{2} \in \operatorname{Lie}_{2}\left(\Lambda^{3} H_{\mathbb{Q}}\right)$ by

$$
\begin{aligned}
& r_{1}:= \begin{cases}{\left[\alpha_{1} \wedge \alpha_{2} \wedge \beta_{2}, \alpha_{3} \wedge \alpha_{4} \wedge \beta_{4}\right]} & \text { if } g \geq 4, \\
0 & \text { if } g=3,\end{cases} \\
& r_{2}:=\left[\alpha_{1} \wedge \alpha_{2} \wedge \beta_{2}, \alpha_{g} \wedge \omega\right] \quad \text { if } g \geq 3 .
\end{aligned}
$$

The following theorem is proved in [12] by completing Hain's arguments [14, §11]:

Proposition 5.3 (Hain, Habegger-Sorger). If $g \geq 6$, then the $\operatorname{Sp}\left(H_{\mathbb{Q}}\right)$-module of quadratic relations $\mathrm{R}_{2}\left(\mathcal{I}_{g, 1}\right)$ is spanned by $r_{1}$ and $r_{2}$.

Actually, Proposition 5.3 and its proof extend to all $g \geq 3$ : See (5.5) below. This proposition together with Hain's result (Theorem 1.1) provides a quadratic presentation of the Torelli Lie algebra in genus $g \geq 6$.

5.3. The Lie bracket $b_{2}$. In order to prove Theorem 1.5, we need to compute the Lie bracket of $\mathcal{A}^{c}\left(H_{\mathbb{Q}}\right)$ in degree $1+1$ :

$$
b_{2}:=[-,-]_{\star}: \Lambda^{2} \mathcal{A}_{1}^{c} \longrightarrow \mathcal{A}_{2}^{c} .
$$

The following formula for $b_{2}$ is deduced from (3.4).

Lemma 5.4. For all $x_{1}, x_{2}, x_{3} \in H_{\mathbb{Q}}$ and $y_{1}, y_{2}, y_{3} \in H_{\mathbb{Q}}$, we have

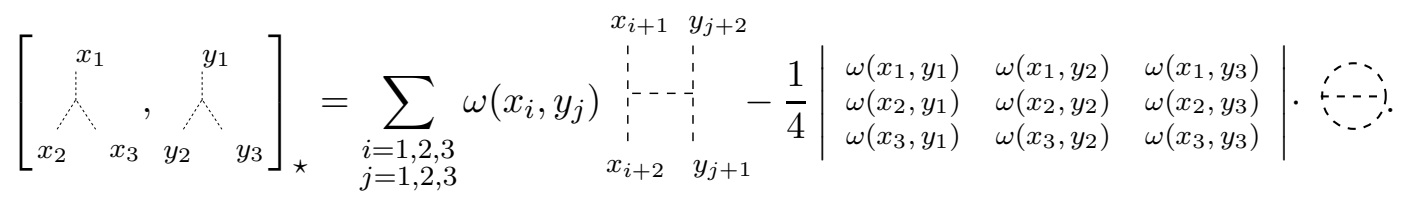

\footnotetext{
${ }^{4}$ When $g=3$, the partition $(2,2,1,1)$ has too many parts to apply directly $(5.1)$. Nevertheless, there is a trick to overpass this restriction: See $[23, \S 6$ (ii)].
} 
The source of the map $b_{2}$ is decomposed into irreducible $\operatorname{Sp}\left(H_{\mathbb{Q}}\right)$-modules according to Lemma 5.2. As for the target of the map $b_{2},(3.5)$ gives the following decomposition:

$$
\mathcal{A}_{2}^{c}=\mathcal{A}_{2,0}^{c} \oplus \mathcal{A}_{2,1}^{c} \oplus \mathcal{A}_{2,2}^{c},
$$

where

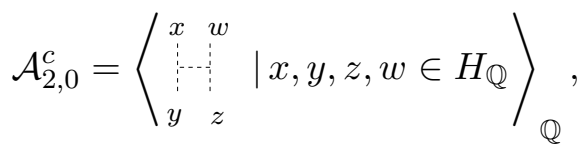

$$
\begin{aligned}
& \mathcal{A}_{2,1}^{c}=\left\langle x^{x} y \quad \mid x, y \in H_{\mathbb{Q}}\right\rangle_{\mathbb{Q}}, \\
& \mathcal{A}_{2,2}^{c}=\langle\cdots\rangle
\end{aligned}
$$

For any $g \geq 0$, we have

$$
\mathcal{A}_{2,0}^{c} \simeq \mathbb{S}_{(2,2)} H_{\mathbb{Q}}, \quad \mathcal{A}_{2,1}^{c} \simeq S^{2} H_{\mathbb{Q}}, \quad \mathcal{A}_{2,2}^{c} \simeq \mathbb{Q} .
$$

So, for $g \geq 3$, we have the following irreducible decompositions into $\operatorname{Sp}\left(H_{\mathbb{Q}}\right)$-modules:

$$
\mathcal{A}_{2,0}^{c} \simeq \Gamma_{0}+\Gamma_{\omega_{2}}+\Gamma_{2 \omega_{2}}, \quad \mathcal{A}_{2,1}^{c} \simeq \Gamma_{2 \omega_{1}}, \quad \mathcal{A}_{2,2}^{c} \simeq \Gamma_{0},
$$

where the decomposition of $\mathcal{A}_{2,0}^{c}$ is deduced from Littlewood's formula (5.1).

5.4. The image of $b_{2} \cdot \operatorname{Im}\left(b_{2}\right)$ is described by the following

Proposition 5.5. If $g \geq 3$, then we have

$$
\operatorname{Im}\left(b_{2}\right)=\mathcal{A}_{2, \mathrm{ev}}^{c}=\mathcal{A}_{2,0}^{c} \oplus \mathcal{A}_{2,2}^{c} \quad\left(\simeq 2 \Gamma_{0}+\Gamma_{\omega_{2}}+\Gamma_{2 \omega_{2}}\right) .
$$

\begin{tabular}{|c|c|}
\hline eigenvalue & eigenvector \\
\hline $2 \omega_{2}$ & $\begin{array}{ccc}\alpha_{2} & \alpha_{1} \\
\vdots & \\
\alpha_{1} & \alpha_{2}\end{array}$ \\
\hline$\omega_{2}$ & $\sum_{i=1}^{g} \underbrace{\alpha_{1} \beta_{i}}_{\alpha_{2} \alpha_{i}}=: \bigwedge_{\alpha_{1}}^{\omega} \bigwedge_{\alpha_{2}}$ \\
\hline 0 & 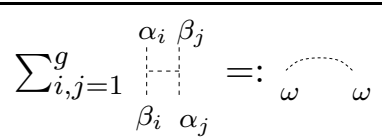 \\
\hline
\end{tabular}

This corresponds to Morita's result $[27,28]$ that $\left(\Gamma_{2} \mathcal{I}_{g, 1} / \Gamma_{3} \mathcal{I}_{g, 1}\right) \otimes \mathbb{Q}$ is classified by the second Johnson homomorphism (onto $\mathcal{A}_{2,0}^{c}$ ) and by the Casson invariant (onto $\mathcal{A}_{2,2}^{c}$ ).

Proof of Proposition 5.5. By (3.6), we have $\operatorname{Im}\left(b_{2}\right) \subset \mathcal{A}_{2, \mathrm{ev}}^{c}$. To prove the converse inclusion, we use decompositions into irreducible $\operatorname{Sp}\left(H_{\mathbb{Q}}\right)$-modules. As mentioned in $[35, \S 3]$, some highest weight vectors of $\mathcal{A}_{2,0}^{c}$ are given by

and this is easily checked. Since

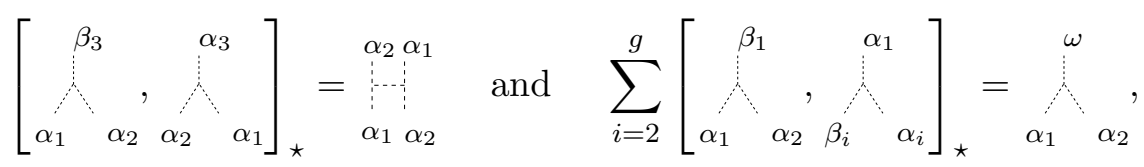

the summand $\Gamma_{2 \omega_{2}}+\Gamma_{\omega_{2}}$ of $\mathcal{A}_{2,0}^{c}$ is in the image of $[-,-]_{\star}$. In order to prove that the summand $2 \Gamma_{0}$ of $\mathcal{A}_{2 \text {,ev }}^{c}$ is in the image as well, we need to compute the Lie bracket of 
the $\operatorname{Sp}\left(H_{\mathbb{Q}}\right)$-invariants of $\Lambda^{2} \mathcal{A}_{1}^{c}$. Those $\operatorname{Sp}\left(H_{\mathbb{Q}}\right)$-invariants of $\Lambda^{2} \Lambda^{3} H_{\mathbb{Q}}$ can be obtained by Morita's method [30, §4.2]: We find

$$
T_{1}:=\sum_{i, j, k=1}^{g} \bigwedge_{\alpha_{j}}^{\alpha_{i}} \wedge \wedge_{\beta_{j}}^{\beta_{i}}
$$

corresponding to the trivalent graph with only one edge and two loop-edges, and

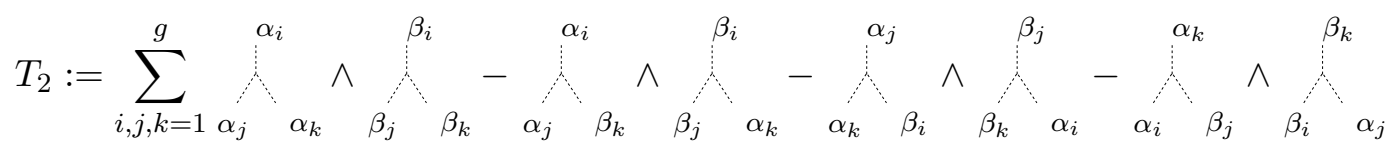

corresponding to the theta-shaped graph. Then, computations by hand lead to

$$
\begin{aligned}
& {\left[T_{1}\right]_{\star}=-\frac{g(g-1)}{4}+(g-1) \omega \omega} \\
& {\left[T_{2}\right]_{\star}=-\frac{g(g-1)(2 g-1)}{2}(-6)+6(g-1) \omega \omega_{\omega} .}
\end{aligned}
$$

Those two vectors of $\mathcal{A}_{2 \text {,ev }}^{c}$ are not colinear since

$$
\left|\begin{array}{cc}
\frac{g(g-1)}{4} & \frac{g(g-1)(2 g-1)}{2} \\
(1-g) & 6(1-g)
\end{array}\right|=g(g-1)^{2}(g-2) \neq 0 .
$$

We deduce that both $\omega_{\omega}$ and are in the image of $[-,-]_{\star}$, thus proving the lemma.

5.5. The kernel of $b_{2}$. $\operatorname{Ker}\left(b_{2}\right)$ is described by the following

Lemma 5.6. If $g \geq 3$, then we have

$$
\operatorname{Ker}\left(b_{2}\right)=\left\langle r_{1}, r_{2}\right\rangle_{\mathrm{Sp}\left(H_{\mathbb{Q}}\right)},
$$

where $r_{1}, r_{2} \in \Lambda^{2} \mathcal{A}_{1}^{c} \simeq \Lambda^{2} \Lambda^{3} H_{\mathbb{Q}}$ are defined in $\S$ 5.2.

Proof. Lemma 5.4 gives $\left[r_{1}\right]_{\star}=0$ and $\left[r_{2}\right]_{\star}=0$ (by the IHX relation). Since the bracket $[-,-]_{\star}$ is $\operatorname{Sp}\left(H_{\mathbb{Q}}\right)$-equivariant, we have

$$
\left\langle r_{1}, r_{2}\right\rangle_{\mathrm{Sp}\left(H_{\mathbb{Q}}\right)} \subset \operatorname{Ker}\left(b_{2}\right) .
$$

Moreover, we have the following inclusions

$$
\left\langle r_{1}, r_{2}\right\rangle_{\mathrm{Sp}\left(H_{\mathbb{Q}}\right)} \supset \begin{cases}2 \Gamma_{\omega_{2}}+\Gamma_{\omega_{1}+\omega_{3}}+2 \Gamma_{\omega_{4}}+\Gamma_{\omega_{2}+\omega_{4}}+\Gamma_{\omega_{6}} & \text { if } g \geq 6 \\ 2 \Gamma_{\omega_{2}}+\Gamma_{\omega_{1}+\omega_{3}}+2 \Gamma_{\omega_{4}}+\Gamma_{\omega_{2}+\omega_{4}} & \text { if } g=5 \\ 2 \Gamma_{\omega_{2}}+\Gamma_{\omega_{1}+\omega_{3}}+\Gamma_{\omega_{4}}+\Gamma_{\omega_{2}+\omega_{4}} & \text { if } g=4 \\ \Gamma_{\omega_{2}}+\Gamma_{\omega_{1}+\omega_{3}} & \text { if } g=3\end{cases}
$$

which is shown in $[12, \S 2]$ for $g \geq 6$ : It is easy to adapt the arguments used there to the cases $g=3,4,5$. Then, it follows from (5.2) and (5.4) that $\left\langle r_{1}, r_{2}\right\rangle_{\mathrm{Sp}\left(H_{\mathbb{Q}}\right)}$ contains a submodule whose complement in $\Lambda^{2} \mathcal{A}_{1}^{c}$ is isomorphic to $2 \Gamma_{0}+\Gamma_{\omega_{2}}+\Gamma_{2 \omega_{2}}$. By Proposition 5.5 , this submodule is isomorphic to $\operatorname{Ker}\left(b_{2}\right)$. Thus, we conclude thanks to (5.3).

We can now complete the proof of Theorem 1.5. Johnson's formula computes the first Johnson homomorphism $\tau_{1}$ on "Bounding Pair" (BP) maps [16, Corollary p.233]. For $i=1,2$, one easily finds a pair of BP maps $f_{i}$ and $g_{i}$ having disjoint supports and 
satisfying $\tau_{1}\left(f_{i}\right) \wedge \tau_{1}\left(g_{i}\right)=r_{i}$. Consequently, the commutative diagram (4.2) gives in degree 2

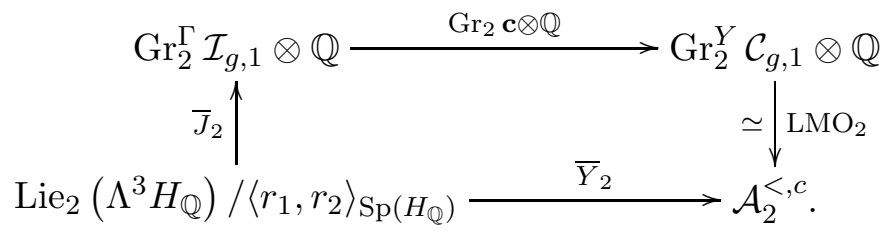

The kernel of $Y_{2}=\chi_{2} \circ b_{2}$ is $\left\langle r_{1}, r_{2}\right\rangle_{\mathrm{Sp}\left(H_{\mathbb{Q}}\right)}$ (by Lemma 5.6), so that the map $\bar{J}_{2}$ in the prevous diagram is injective. Thus, we obtain

$$
\mathrm{R}_{2}\left(\mathcal{I}_{g, 1}\right)=\left\langle r_{1}, r_{2}\right\rangle_{\mathrm{Sp}\left(H_{\mathbb{Q}}\right)} \quad \text { for } g \geq 3
$$

which slightly generalizes Proposition 5.3. We conclude that $\operatorname{Ker}\left(Y_{2}\right)=\mathrm{R}_{2}\left(\mathcal{I}_{g, 1}\right)$.

\section{Stability with Respect to the Genus}

In this short section, we consider stability with respect to the genus for the filtrations $\Gamma$ and $Y$ on $\mathcal{I}_{g, 1}$ and $\mathcal{C}_{g, 1}$, respectively. We start by fixing surface inclusions

$$
\Sigma_{0,1} \subset \Sigma_{1,1} \subset \Sigma_{2,1} \subset \cdots .
$$

Thus, we have group monomorphisms

$$
\mathcal{I}_{0,1} \hookrightarrow \mathcal{I}_{1,1} \hookrightarrow \mathcal{I}_{2,1} \hookrightarrow \cdots,
$$

which allows us to regard $\mathcal{I}_{g, 1}$ as a subgroup of $\lim _{g} \mathcal{I}_{g, 1}$. Then, the stabilized lower central series of $\mathcal{I}_{g, 1}$ is defined by

$$
\Gamma_{i}^{\mathrm{stab}} \mathcal{I}_{g, 1}:=\mathcal{I}_{g, 1} \cap \Gamma_{i} \underset{g}{\lim } \mathcal{I}_{g, 1}=\mathcal{I}_{g, 1} \cap \underset{g}{\lim } \Gamma_{i} \mathcal{I}_{g, 1} .
$$

Similarly, we can regard $\mathcal{C}_{g, 1}$ as a submonoid of $\lim _{g} \mathcal{C}_{g, 1}$. The stabilized $Y$-filtration of $\mathcal{C}_{g, 1}$ is defined by

$$
Y_{i}^{\text {stab }} \mathcal{C}_{g, 1}:=\mathcal{C}_{g, 1} \cap \underset{g}{\lim } Y_{i} \mathcal{C}_{g, 1}
$$

The following proposition means that the $Y$-filtration for homology cylinders is stable.

Proposition 6.1. For all $g \geq 0$ and for all $i \geq 1$, we have

$$
Y_{i} \mathcal{C}_{g, 1}=Y_{i}^{\mathrm{stab}} \mathcal{C}_{g, 1}
$$

Proposition 6.1 is proved at the end of this section, and we now use it to state Conjecture 1.7 in a different way:

Conjecture 6.2. For any $g \geq 0$, the stabilized lower central series of the Torelli group $\mathcal{I}_{g, 1}$ coincides with the $Y$-filtration:

$$
\Gamma_{j}^{\mathrm{stab}} \mathcal{I}_{g, 1}=\mathcal{I}_{g, 1} \cap Y_{j} \mathcal{C}_{g, 1} \quad \text { for all } j \geq 1 .
$$

The inclusion " $\subset$ " in (6.1) holds true, and it can be deduced from Proposition 6.1 as follows. If $x \in \Gamma_{j}^{\mathrm{stab}} \mathcal{I}_{g, 1}$, then $x \in \mathcal{I}_{g, 1}$ and $x \in \Gamma_{j} \mathcal{I}_{g^{\prime}, 1}$ for some $g^{\prime} \geq g$. The latter implies that $x \in Y_{j} \mathcal{C}_{g^{\prime}, 1}$ and, since $x \in \mathcal{C}_{g, 1}$, we deduce that $x \in Y_{j}^{\text {stab }} \mathcal{C}_{g, 1}=Y_{j} \mathcal{C}_{g, 1}$. 
Proof that Conjectures 1.7 and 6.2 are equivalent. Conjecture 1.7 is equivalent to the injectivity of

$$
\underset{g}{\lim } \operatorname{Gr}_{i} \mathbf{c}: \underset{g}{\lim _{\mathrm{g}}} \Gamma_{i} \mathcal{I}_{g, 1} / \Gamma_{i+1} \mathcal{I}_{g, 1} \longrightarrow \underset{g}{\longrightarrow} \lim _{i} \mathcal{C}_{g, 1} / Y_{i+1}
$$

for all $i \geq 1$. By Proposition 6.1, injectivity of (6.2) is equivalent to the statement that, if $x \in \Gamma_{i} \mathcal{I}_{g, 1}$ satisfies $x \in Y_{i+1} \mathcal{C}_{g, 1}$ then $x \in \Gamma_{i+1}^{\text {stab }} \mathcal{I}_{g, 1}$. So, Conjecture 1.7 is equivalent to the statement that

$$
\Gamma_{i} \mathcal{I}_{g, 1} \cap Y_{i+1} \mathcal{C}_{g, 1} \subset \Gamma_{i+1}^{\mathrm{stab}} \mathcal{I}_{g, 1} \quad \text { for all } i \geq 1 \text {. }
$$

Clearly, the inclusion " $\supset$ " in (6.1) implies (6.3). Conversely, using Proposition 6.1 again, it is easily shown by induction on $j \geq 1$ that (6.3) implies the inclusion " $\supset$ " in (6.1). Thus Conjecture 1.7 is equivalent to Conjecture 6.2.

Proof of Proposition 6.1. The inclusion " $\subset$ " is obvious. To prove " $\supset$ ", suppose that $x \in Y_{i}^{\text {stab }} \mathcal{C}_{g, 1}$ is represented by a homology cylinder $M$ over $\Sigma_{g, 1}$. Then we have $x \in \mathcal{C}_{g, 1}$ and $x \in Y_{i} \mathcal{C}_{g^{\prime}, 1}$ for some $g^{\prime}>g$. In other words, the homology cylinder

$$
M^{\prime}=M \cup_{\partial \Sigma_{g, 1} \times[-1,1]}\left(\left(\Sigma_{g^{\prime}, 1} \backslash \operatorname{int} \Sigma_{g, 1}\right) \times[-1,1]\right)
$$

over $\Sigma_{g^{\prime}, 1}$, which represents $x \in Y_{i} \mathcal{C}_{g^{\prime}, 1}$, is $Y_{i}$-equivalent to the trivial cylinder $M_{0}:=$ $\Sigma_{g^{\prime}, 1} \times[-1,1]$. Hence there are mutually disjoint, connected graph claspers $C_{1}, \ldots, C_{p}$ $(p \geq 0)$ in $M_{0}$, each having $i$ nodes, such that the result $\left(M_{0}\right)_{C_{1}, \ldots, C_{p}}$ from $M_{0}$ of surgery along $C_{1}, \ldots, C_{p}$ is homeomorphic to $M^{\prime}$ relative to boundary.

Let $\tilde{\Sigma}_{g, 1}$ denote a surface obtained from $\Sigma_{g, 1}$ by attaching a collar $N:=S^{1} \times[0,1]$ along $\partial \Sigma_{g, 1}$. Thus $\tilde{\Sigma}_{g, 1}$ is a compact, oriented surface of genus $g$ with one boundary component, which contain $\Sigma_{g, 1}$ in its interior. There is a (not proper) embedding

$$
f: \Sigma_{g^{\prime}, 1} \times[-1,1] \hookrightarrow \tilde{\Sigma}_{g, 1} \times[-1,1]
$$

such that

- $f$ is the identity on $\Sigma_{g, 1} \times[-1,1]$,

- $f$ maps $\partial \Sigma_{g^{\prime}, 1} \times[-1,1]$ homeomorphically onto $\partial \tilde{\Sigma}_{g, 1} \times[-1,1]$.

Let $M^{\prime \prime}:=\left(\tilde{\Sigma}_{g, 1} \times[-1,1]\right)_{f\left(C_{1}\right), \ldots, f\left(C_{p}\right)}$ be the homology cylinder over $\tilde{\Sigma}_{g, 1}$ obtained from the cylinder $\tilde{\Sigma}_{g, 1} \times[-1,1]$ by surgery along $f\left(C_{1}\right), \ldots, f\left(C_{p}\right)$. If we regard $M^{\prime \prime}$ as a homology cylinder over $\Sigma_{g, 1}$ by a homeomorphism $\tilde{\Sigma}_{g, 1} \cong \Sigma_{g, 1}$ which is identity outside a small neighborhood of the collar $N \subset \tilde{\Sigma}_{g, 1}$, then $M^{\prime \prime}$ is homeomorphic to $M$ relative to boundary. Hence $M$ is $Y_{i}$-equivalent to the trivial cylinder, i.e. $x \in Y_{i} \mathcal{C}_{g, 1}$.

\section{The Closed surface CASE}

In this section, we extend our results to the case of a closed connected oriented surface of genus $g$, which we denote by $\Sigma_{g}$. We set $H:=H_{1}\left(\Sigma_{g}\right)$ and $\omega: H \otimes H \rightarrow \mathbb{Z}$ denotes the intersection pairing of $\Sigma_{g}$. Let $\mathcal{I}_{g}$ be the Torelli group of the surface $\Sigma_{g}$, and let $\mathcal{C}_{g}$ be the monoid of homology cylinders over $\Sigma_{g}$. 
7.1. The ideals $I^{<} \subset \mathcal{A}^{<}$and $I^{<, c} \subset \mathcal{A}^{<, c}$. In this subsection, we introduce an ideal $I^{<}$in the algebra $\mathcal{A}^{<}$and an ideal $I^{<, c}$ in the Lie algebra $\mathcal{A}^{<, c}$. The latter appears in [13].

By an $\omega$-diagram, we mean a Jacobi diagram such that each external vertex is labeled by either an element of $H_{\mathbb{Q}}$ or the symbol " $\omega$ ", and such that each component has at least one internal vertex or has at least one vertex labeled $\omega$. An external vertex labeled $\omega$ is called an $\omega$-vertex. The degree of an $\omega$-diagram $D$ is defined to be the sum of the internal degree of $D$ and the number of $\omega$-vertices in $D$.

By an $(\omega,<)$-diagram, we mean an $\omega$-diagram whose external vertices are totally ordered. To each $(\omega,<)$-diagram $D$, we associate an element in $\mathcal{A}^{<}$as follows:

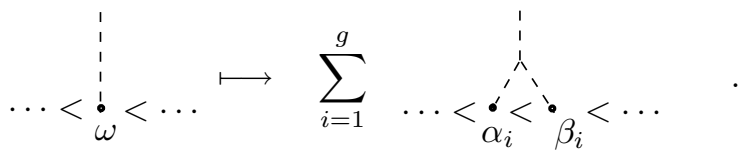

Using this rule, we regard $(\omega,<)$-diagrams as elements of $\mathcal{A}^{<}$. Some basic properties for $(\omega,<)$-diagrams are in order.

Lemma 7.1 (STU-relation for $\omega$-vertex). In the space $\mathcal{A}^{<}$, we have the following identities:

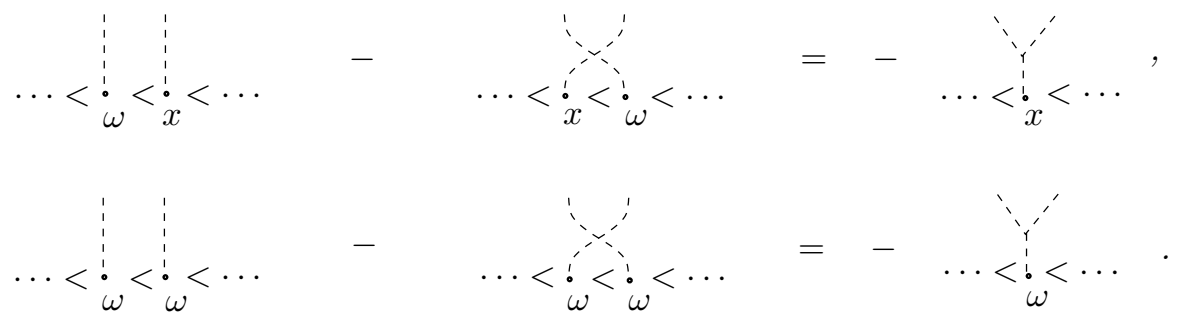

Proof. The first identity follows for $2 g$ applications of the STU-like relation in the space $\mathcal{A}^{<}$. The second identity is proved by applying $2 g$ times the first identity and using the IHX relation.

Lemma 7.2 (Commutation identity for $\omega$-vertex). In the space $\mathcal{A}^{<}$, an $\omega$-vertex commutes with any $(\omega,<)$-diagram, i.e.

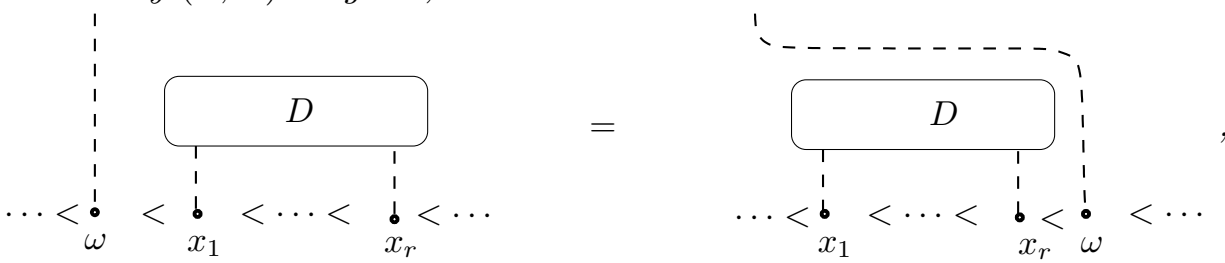

where $x_{1}, \ldots, x_{r}$ belong to $H_{\mathbb{Q}} \cup\{\omega\}$.

Proof. According to Lemma 7.1, the difference between the left hand side term and the right hand side term is given by

$$
d:=-\begin{gathered}
1 \\
\hdashline \\
\hdashline \\
\dot{x}_{1}<\cdots<\dot{x}_{r}
\end{gathered},
$$

where the "box" notation is recalled in Figure 7.1. Using the IHX and AS relations, one can "slide" the box upwards which shows that $d=0$. 

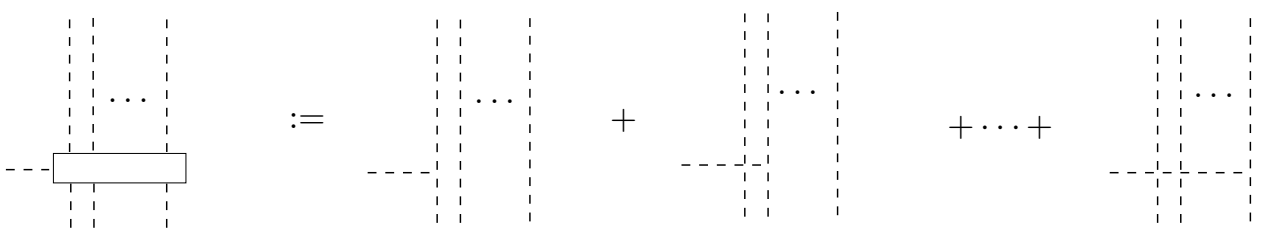

FIGURE 7.1. The box notation

Lemma 7.3. $\operatorname{Sp}\left(H_{\mathbb{Q}}\right)$ acts trivially on each $\omega$-vertex in a $(\omega,<)$-diagram. That is, if $D$ is an $(\omega,<)$-diagram with some $\omega$-vertices and other vertices $u_{1}, \ldots, u_{l}$ labeled by $c_{1}, \ldots, c_{l} \in H_{\mathbb{Q}}$ respectively, then, for each $F \in \operatorname{Sp}\left(H_{\mathbb{Q}}\right), F \cdot D \in \mathcal{A}^{<}$is represented by the diagram obtained from $D$ by changing the labels for $u_{1}, \ldots, u_{l}$ with $F\left(c_{1}\right), \ldots, F\left(c_{l}\right)$ respectively and by leaving the $\omega$-vertices unchanged.

Proof. This is immediate from the fact that $F \cdot \omega=\omega \in \Lambda^{2} H_{\mathbb{Q}}$ for all $F \in \operatorname{Sp}\left(H_{\mathbb{Q}}\right)$.

An $(\omega,<)$-diagram is called an $\omega$-smallest diagram if the smallest external vertex is an $\omega$-vertex. Let $I^{<} \subset \mathcal{A}^{<}$denote the (homogeneous) subspace spanned by $\omega$-smallest diagrams.

Proposition 7.4. $I^{<}$is a Hopf ideal in $\mathcal{A}^{<}$, closed under the $\operatorname{Sp}\left(H_{\mathbb{Q}}\right)$-action.

Proof of Proposition 7.4. It is clear that $I^{<}$is a right ideal in the algebra $\mathcal{A}^{<}$. Lemma 7.2 implies that $I^{<}$is a left ideal in $\mathcal{A}^{<}$as well. Thus $I^{<}$is a two-sided ideal. It is easy to check that $I^{<}$is a Hopf ideal. By Lemma 7.3, $I^{<}$is closed under the $\operatorname{Sp}\left(H_{\mathbb{Q}}\right)$-action.

Therefore, the quotient $\mathcal{A}^{<} / I^{<}$is a graded Hopf algebra with $\operatorname{Sp}\left(H_{\mathbb{Q}}\right)$-action. It also follows from Proposition 7.4 that

$$
I^{<, c}:=I^{<} \cap \mathcal{A}^{<, c}
$$

is an ideal in the Lie algebra $\mathcal{A}^{<, c}$. Using the fact that $\mathcal{A}^{<}=U\left(\mathcal{A}^{<, c}\right)$, it can be seen that the subspace $I^{<, c}$ is spanned by connected $\omega$-smallest diagrams.

7.2. Diagrammatic description of $\operatorname{Gr}^{Y} \mathcal{C}_{g} \otimes \mathbb{Q}$. We can now state the main result of this section, which is the analogue of Theorem 1.2 in the closed surface case.

To relate the bordered surface case to the closed surface case, we fix an embedding

$$
\text { i : } \Sigma_{g, 1} \hookrightarrow \Sigma_{g},
$$

which induces a surjective homomorphism of graded Lie algebras with $\operatorname{Sp}\left(H_{\mathbb{Q}}\right)$-action:

$$
\operatorname{Gr} \mathbf{i} \otimes \mathbb{Q}: \operatorname{Gr}^{Y} \mathcal{C}_{g, 1} \otimes \mathbb{Q} \longrightarrow \operatorname{Gr}^{Y} \mathcal{C}_{g} \otimes \mathbb{Q} .
$$

Theorem 7.5. Let $g \geq 0$. There exist mutually inverse isomorphisms of graded Lie algebras with $\mathrm{Sp}\left(H_{\mathbb{Q}}\right)$-action

$$
\mathcal{A}^{<, c}\left(H_{\mathbb{Q}}\right) / I^{<, c}\left(H_{\mathbb{Q}}\right) \stackrel{\psi}{\rightleftarrows} \operatorname{Gr}^{Y} \mathcal{C}_{g} \otimes \mathbb{Q}
$$

such that the following diagram is commutative:

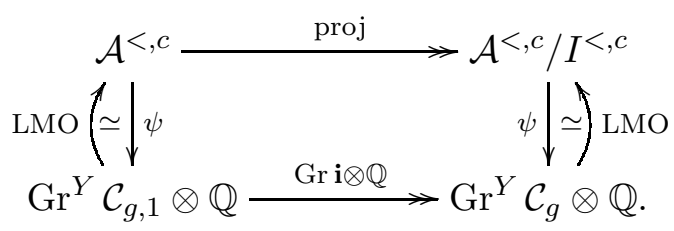


The proof of Theorem 7.5 is given in $\S 7.3$ and $\S 7.4$.

Remark 7.6. The definition of the surgery map $\psi: \mathcal{A}^{<, c} / I^{<, c} \longrightarrow \operatorname{Gr}^{Y} \mathcal{C}_{g} \otimes \mathbb{Q}$ is suggested in [13], where it is also conjectured to be an isomorphism. The degree 1 case is done in $[25]$.

7.3. The surgery map $\psi$. Let $\psi: \mathcal{A}^{<, c} \rightarrow \operatorname{Gr}^{Y} \mathcal{C}_{g, 1} \otimes \mathbb{Q}$ be the surgery map defined in $\S 2.2$ in the case of the bordered surface $\Sigma_{g, 1}$.

Lemma 7.7. The map $(\mathrm{Gr} \mathbf{i} \otimes \mathbb{Q}) \circ \psi$ vanishes on the subspace $I^{<, c}$.

Consequently, the surgery map for $\Sigma_{g, 1}$ induces a surgery map for $\Sigma_{g}$

$$
\psi: \mathcal{A}^{<, c} / I^{<, c} \longrightarrow \operatorname{Gr}^{Y} \mathcal{C}_{g} \otimes \mathbb{Q}
$$

such that $\psi \circ \operatorname{proj}=(\operatorname{Gr} \mathbf{i} \otimes \mathbb{Q}) \circ \psi$.

Proof of Lemma 7.7. Let $D$ be a connected $\omega$-smallest diagram of degree $i$. By the rule (7.1), we can assume that $D$ has only one $\omega$-vertex $v$, and it suffices to show that $(\mathrm{Gr} \mathbf{i} \otimes \mathbb{Q}) \circ \psi(D)=0$. Let $C$ be a connected graph clasper in $\Sigma_{g, 1} \times[-1,1]$, which realizes "topologically" $D$ in the sense of $\S 2.2$, except that the leaf $L_{v}$ corresponding to $v$ goes along $\partial \Sigma_{g, 1} \times\{t\}$, where $t \in(-1,1)$.

The graph clasper $C$ can be regarded in the product $\Sigma_{g} \times[-1,1]$ via the inclusion i : $\Sigma_{g, 1} \hookrightarrow \Sigma_{g}$. There, the leaf $L_{v}$ bounds a disk whose interior does not intersect $C$. Therefore, we have

$$
\left(\Sigma_{g} \times[-1,1]\right)_{\mathbf{i}(C)} \cong \Sigma_{g} \times[-1,1] .
$$

Since $v$ is the smallest external vertex, one can arrange by an isotopy of $C$ that the level surface $\Sigma_{g, 1} \times\{t\}$, bounded by the leaf $L_{v}$, does not intersect the edges of $C$. So, by clasper calculus [13], the graph clasper $C$ can be transformed to a clasper $C^{\prime}$ by trading the leaf $L_{v}$ for a box $B$ with $g$ input edges $e_{1}, \ldots, e_{g}$ such that each $e_{j}$ is connected to a node $w_{j}$, which is itself connected by edges to two leaves representing the isotopy classes of the curves $\alpha_{j}$ and $\beta_{j}$, respectively. Recall that $\left(\alpha_{1}, \beta_{1}, \ldots, \alpha_{g}, \beta_{g}\right)$ is a system of meridians and parallels for the surface $\Sigma_{g, 1}$, as shown on Figure 4.1.

Next, by using the zip construction, we see that surgery along $C^{\prime}$ is $Y_{i+1}$-equivalent to surgery along the disjoint union of $g$ graph claspers $C_{1}, \ldots, C_{g}$, where $C_{j}$ is obtained from $C$ by replacing the leaf $L_{v}$ with a node connected by edges to two leaves representing $\alpha_{j}$ and $\beta_{j}$, respectively.

Let $D_{1}, \ldots, D_{g}$ denote the $(\omega,<)$-diagrams obtained from $D$ by replacing $v$ with an internal vertex connected to two external vertices labeled by $\alpha_{i}$ and $\beta_{i}$, respectively, the former being declared smaller than the latter. Then we have

$$
\psi\left(\sum_{j=1}^{g} D_{j}\right)= \pm \sum_{j=1}^{g}\left\{\left(\Sigma_{g, 1} \times[-1,1]\right)_{C_{j}}\right\}_{Y_{i+1}}= \pm\left\{\left(\Sigma_{g, 1} \times[-1,1]\right)_{C^{\prime}}\right\}_{Y_{i+1}},
$$

where the second identity is proved by clasper calculus. By (7.5), $\operatorname{Gr} \mathbf{i} \otimes \mathbb{Q}$ maps the right hand side to 0 . Hence we have

$$
(\mathrm{Gr} \mathbf{i} \otimes \mathbb{Q}) \circ \psi(D)=(\operatorname{Gr} \mathbf{i} \otimes \mathbb{Q}) \circ \psi\left(\sum_{i=1}^{g} D_{i}\right)=0 .
$$


7.4. The LMO map. In this subsection, we construct a Lie algebra homomorphism

$$
\text { LMO: } \operatorname{Gr}^{Y} \mathcal{C}_{g} \otimes \mathbb{Q} \longrightarrow \mathcal{A}^{<, c} / I^{<, c} \text {. }
$$

It is induced by the map LMO : $\operatorname{Gr}^{Y} \mathcal{C}_{g, 1} \otimes \mathbb{Q} \longrightarrow \mathcal{A}^{<, c}$ of $\S 2.3$, in the sense that $\mathrm{LMO} \circ(\mathrm{Gr} \mathbf{i} \otimes \mathbb{Q})=\operatorname{proj} \circ \mathrm{LMO}$. This will complete the proof of Theorem 7.5.

To do this, it is enough to show that the monoid homomorphism LMO $=s \circ \varphi \circ \widetilde{Z}^{Y}$ : $\mathcal{C}_{g, 1} \rightarrow \mathcal{A}^{<}$introduced in $\S 2.3$ induces a monoid homomorphism

$$
\mathrm{LMO}: \mathcal{C}_{g} \longrightarrow \mathcal{A}^{<} / I^{<}
$$

We start by recalling from [4] how the maps $\widetilde{Z}^{Y}$ and $s$ are defined. First of all, a LMO functor

$$
\widetilde{Z}: \mathcal{L C} o b \longrightarrow{ }^{t s} \mathcal{A}
$$

is defined from the category $\mathcal{L C} o b$ of "Lagrangian cobordisms" between compact connected oriented surfaces with circle boundary ${ }^{5}$ to the category ${ }^{t} \mathcal{A}$ of "top-substantial Jacobi diagrams". The category ${ }^{t s} \mathcal{A}$ has non-negative integers as objects. For $g, h \geq 0$, ${ }^{t s} \mathcal{A}(g, f)$ is (the degree completion of) a $\mathbb{Q}$-vector space spanned by Jacobi diagrams with external vertices labelled by $\lfloor g\rceil^{+}:=\left\{1^{+}, \ldots, g^{+}\right\}$or $\lfloor f\rceil^{-}:=\left\{1^{-}, \ldots, f^{-}\right\}$such that each strut component, if any, does not connect an $i^{+}$with a $j^{+}$, for some $1 \leq i, j \leq g$. The identity of $g \geq 0$ in the category ${ }^{t s} \mathcal{A}$ is the following exponential of struts:

$$
\operatorname{Id}_{g}=\exp _{\sqcup}\left(\sum_{i=1}^{g} i_{i^{-}}^{i^{+}}\right) .
$$

Since $\mathcal{L} \mathcal{C} o b(g, g)$ contains the monoid $\mathcal{C}_{g, 1}$ of homology cylinders, $\widetilde{Z}$ restricts to an invariant of homology cylinders. Let $\mathcal{A}^{Y}\left(\lfloor g\rceil^{+} \cup\lfloor g\rceil^{-}\right)$be the space of Jacobi diagrams with external vertices colored by $\lfloor g\rceil^{+} \cup\lfloor g\rceil^{-}$and without strut component, modulo the AS, IHX and multilinearity relations. It is shown in [4] that, for all $M \in \mathcal{C}_{g, 1}$, $\widetilde{Z}(M) \in{ }^{t s} \mathcal{A}(g, g)$ splits as

$$
\widetilde{Z}(M)=\operatorname{Id}_{g} \sqcup \widetilde{Z}^{Y}(M)
$$

where

$$
\widetilde{Z}^{Y}(M) \in \mathcal{A}^{Y}\left(\lfloor g\rceil^{+} \cup\lfloor g\rceil^{-}\right)
$$

denotes the $Y$-reduction of $\widetilde{Z}(M)$. Thus, we obtain a monoid homomorphism

$$
\widetilde{Z}^{Y}: \mathcal{C}_{g, 1} \longrightarrow \mathcal{A}^{Y}\left(\lfloor g\rceil^{+} \cup\lfloor g\rceil^{-}\right),
$$

where the space $\mathcal{A}^{Y}\left(\lfloor g\rceil^{+} \cup\lfloor g\rceil^{-}\right)$is equipped with the multiplication $\star$ defined by

$$
D \star E:=\left(\begin{array}{c}
\text { sum of all ways of gluing some of the } i^{+} \text {-colored vertices of } D \\
\text { to some of the } i^{-} \text {-colored vertices of } E \text {, for all } i=1, \ldots, g
\end{array}\right) \text {. }
$$

This product was introduced in [8].

As for the graded algebra isomorphism

$$
\varphi:\left(\mathcal{A}^{Y}\left(\lfloor g\rceil^{+} \cup\lfloor g\rceil^{-}\right), \star\right) \longrightarrow\left(\mathcal{A}^{<}\left(-H_{\mathbb{Q}}\right), \stackrel{\sqcup}{\sqcup}\right)
$$

it is defined by declaring that "each $i^{-}$-colored vertex should be smaller than any $i^{+}$colored vertex" and by changing the colors of external vertices according to the rules $\left(i^{-} \mapsto \alpha_{i}\right)$ and $\left(i^{+} \mapsto \beta_{i}\right)$. See [4].

\footnotetext{
${ }^{5}$ To be exact, one has to choose a parenthesizing of the handles of the surface $\Sigma_{g, 1}$ for each $g \geq 0$.
} 
Instead of considering cobordisms between surfaces with one boundary component, one can consider cobordisms between closed surfaces. We defined in [4] a congruence relation $\sim$ on $\mathcal{L C} o b$ such that the quotient category $\mathcal{L C o b} / \sim$ gives the category of "Lagrangian cobordisms" between closed surfaces.

For $g \geq 0$, let $a_{g} \in{ }^{t s} \mathcal{A}(g+1, g)$ be defined by

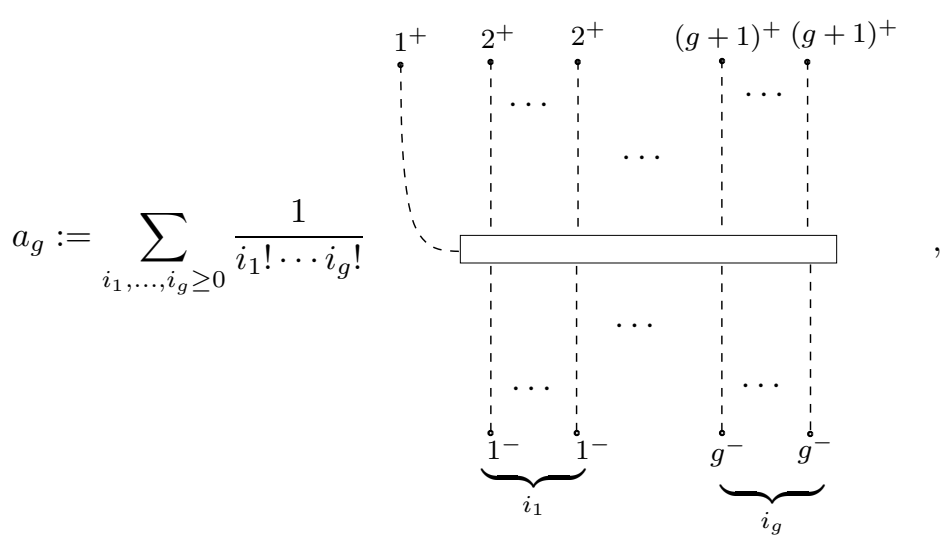

where the "box" notation is recalled in Figure 7.1. For $g, f \geq 0$, let $\mathcal{I}(g, f)$ denote the subspace of ${ }^{t s} \mathcal{A}(g, f)$ defined by

$$
\mathcal{I}(g, f)=\left\{a_{f} \circ x \mid x \in{ }^{t s} \mathcal{A}(g, f+1)\right\} .
$$

The vector spaces $\mathcal{I}(g, f)$, where $g, f \geq 0$, form an ideal in the linear category ${ }^{t s} \mathcal{A}$, i.e., we have

$$
\begin{aligned}
& { }^{t s} \mathcal{A}(g, f) \circ \mathcal{I}(h, g) \subset \mathcal{I}(h, f), \\
& \mathcal{I}(g, f) \circ{ }^{t s} \mathcal{A}(h, g) \subset \mathcal{I}(h, f) .
\end{aligned}
$$

Thus, we can consider the quotient category ${ }^{t s} \mathcal{A} / \mathcal{I}$. The ideal $\mathcal{I}$ of ${ }^{t s} \mathcal{A}$ is introduced in an equivalent way in [4], where it is proved that the LMO functor sends $\sim$ to the congruence relation defined by $\mathcal{I}$. Hence a functor on the category of Lagrangian cobordisms between closed surfaces:

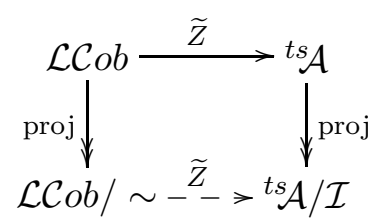

We are going to prove the following technical result.

Lemma 7.8. Each element $y$ of $\mathcal{A}^{Y}\left(\lfloor g\rceil^{+} \cup\lfloor g\rceil^{-}\right)$, such that $y \sqcup \operatorname{Id}_{g}$ belongs to $\mathcal{I}(g, g)$, is sent by the algebra map $s \circ \varphi$ to an element of $I^{<}\left(H_{\mathbb{Q}}\right) \subset \mathcal{A}^{<}\left(H_{\mathbb{Q}}\right)$.

This lemma shows that LMO $=s \circ \varphi \circ \widetilde{Z}^{Y}: \mathcal{C}_{g, 1} \rightarrow \mathcal{A}^{<}$induces a monoid homomorphism LMO : $\mathcal{C}_{g} \rightarrow \mathcal{A}^{<} / I^{<}$as desired. Indeed, assume that $M, M^{\prime} \in \mathcal{C}_{g, 1}$ are such that $\mathbf{i}(M)=\mathbf{i}\left(M^{\prime}\right) \in \mathcal{C}_{g}$. Then, the Lagrangian cobordisms $M$ and $M^{\prime}$ are congruent so that $\widetilde{Z}(M)-\widetilde{Z}\left(M^{\prime}\right) \in \mathcal{I}(g, g)$. Lemma 7.8 implies that

$$
\operatorname{LMO}(M)-\operatorname{LMO}\left(M^{\prime}\right)=s \circ \varphi\left(\widetilde{Z}^{Y}(M)-\widetilde{Z}^{Y}\left(M^{\prime}\right)\right) \in I^{<} .
$$

This will conclude the proof of Theorem 7.5. 
Proof of Lemma 7.8. Let $y \in \mathcal{A}^{Y}\left(\lfloor g\rceil^{+} \cup\lfloor g\rceil^{-}\right)$be such that

$$
t:=y \sqcup \exp _{\sqcup}\left(\sum_{i=1}^{g} i_{i^{-}}^{i^{+}}\right) \in \mathcal{I}(g, g) .
$$

Then, $t$ is a series of elements of the form

$$
u_{x}:=a_{g} \circ\left(x \sqcup \exp _{\sqcup}\left(\sum_{i=1}^{g} i_{(i+1)^{-}}^{i^{+}}\right)\right),
$$

where either

(1) $x \in \mathcal{A}^{Y}\left(\lfloor g\rceil^{+} \cup\lfloor g+1\rceil^{-}\right)$is a diagram involving exactly one label $1^{-}$, or

(2) $x=x^{\prime} \sqcup x^{\prime \prime}$ with $x^{\prime} \in \mathcal{A}^{Y}\left(\lfloor g\rceil^{+} \cup\lfloor g+1\rceil^{-}\right)$involving no label $1^{-}$, and $x^{\prime \prime}=e^{+}$ with $e \in\{1, \ldots, g\}$ or $x^{\prime \prime}=1^{-} e^{-}$with $e \in\{2, \ldots, g+1\}$.

Then, we have

$$
u_{x}=u_{x}^{Y} \sqcup \exp _{\sqcup}\left(\sum_{i=1}^{g} i^{i^{+}}\right),
$$

where

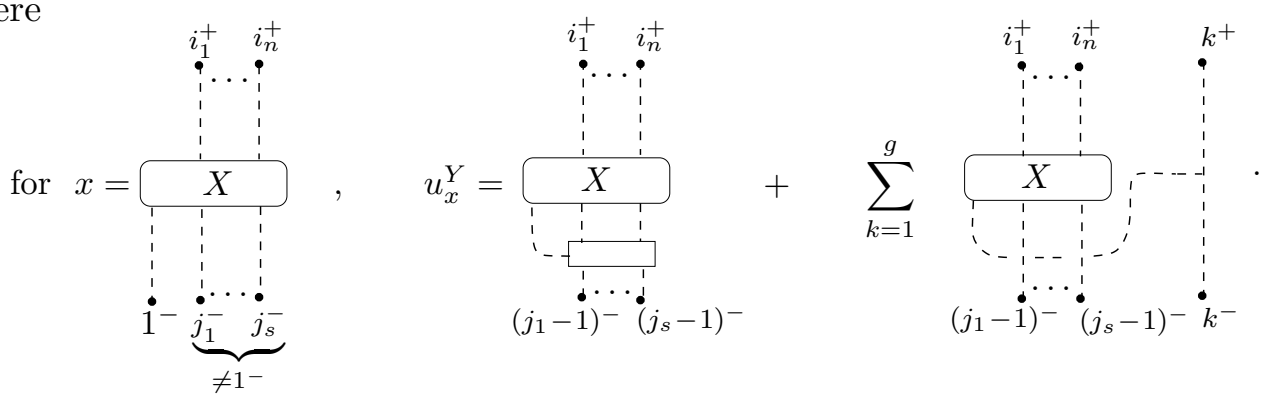

So, it is enough to show that, for all $x$ of the above form, $s \circ \varphi\left(u_{x}^{Y}\right)$ is an $\omega$-smallest diagram. This follows from Lemma 7.1.

Remark 7.9. The surgery map $\psi: \mathcal{A}^{<, c} / I^{<, c} \rightarrow \operatorname{Gr}^{Y} \mathcal{C}_{g} \otimes \mathbb{Q}$ does not depend on the choice (7.2) of the embedding $\mathbf{i}: \Sigma_{g, 1} \hookrightarrow \Sigma_{g}$ because, by clasper calculus, it can be defined directly with no reference to $\Sigma_{g, 1}$. Consequently, LMO $=\psi^{-1}: \operatorname{Gr}^{Y} \mathcal{C}_{g} \otimes \mathbb{Q} \rightarrow \mathcal{A}^{<, c} / I^{<, c}$ is independent of $\mathbf{i}$ too. But, the monoid homomorphism LMO $: \mathcal{C}_{g} \rightarrow \mathcal{A}^{<} / I^{<}$does depend on the choices of $\mathbf{i}$ and $\left(\alpha_{1}, \ldots, \alpha_{g}, \beta_{1}, \ldots, \beta_{g}\right)$, see Remark 4.3.

7.5. The ideals $I \subset \mathcal{A}$ and $I^{c} \subset \mathcal{A}^{c}$. We defined in $\S 3.1$ an $\operatorname{Sp}\left(H_{\mathbb{Q}}\right)$-equivariant, graded Hopf algebra isomorphism

$$
\chi: \mathcal{A} \stackrel{\simeq}{\longrightarrow} \mathcal{A}^{<},
$$

which restricts to an $\operatorname{Sp}\left(H_{\mathbb{Q}}\right)$-equivariant, graded Lie algebra isomorphism

$$
\chi: \mathcal{A}^{c} \stackrel{\simeq}{\longrightarrow} \mathcal{A}^{<, c} .
$$

The purpose of this subsection is to compute

$$
I:=\chi^{-1}\left(I^{<}\right) \subset \mathcal{A}, \quad I^{c}:=\chi^{-1}\left(I^{<, c}\right) \subset \mathcal{A}^{c},
$$

which are ideals in $\mathcal{A}$ and $\mathcal{A}^{c}$, respectively, closed under the $\operatorname{Sp}\left(H_{\mathbb{Q}}\right)$-action. 
Proposition 7.10. The subspace $I$ of $\mathcal{A}$ is spanned by elements of the form

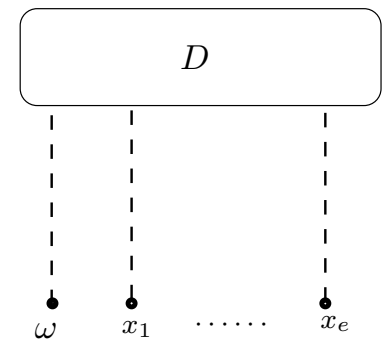

$$
-\frac{1}{4} \sum_{1 \leq j<k \leq e} \omega\left(x_{j}, x_{k}\right)
$$

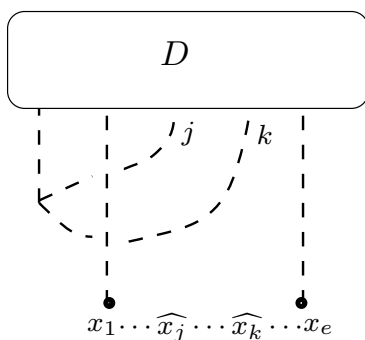

where $x_{1} \ldots, x_{e} \in H_{\mathbb{Q}}$ and where the $\omega$-vertex means

$$
=\sum_{i=1}^{g} \stackrel{1}{1}
$$

Moreover, the subspace $I^{c}$ of $\mathcal{A}^{c}$ is spanned by elements of the form (7.9) where $D$ is assumed to be connected.

Proof. Let $\check{I}$ denote the $\mathbb{Q}$-vector space spanned by $\omega$-diagrams with exactly one $\omega$ vertex, modulo the AS, IHX and multilinearity relations. Similarly, let $\check{I}^{<}$denote the $\mathbb{Q}$-vector space spanned by $\omega$-smallest diagrams with exactly one $\omega$-vertex, modulo the AS, IHX, STU-like and multilinearity relations. (Here, the STU-like relation is not applicable to the $\omega$-vertex.) There is a natural surjective linear map

$$
\lambda^{<}: \check{I}^{<} \longrightarrow I^{<} \text {, }
$$

which maps each diagram in $\check{I}^{<}$to itself, using the rule (7.1).

We consider the linear map

$$
k: \check{I} \longrightarrow \check{I}^{<},
$$

that maps each diagram $D \in \check{I}$ with $e H_{\mathbb{Q}^{-c o l o r e d ~}}$ external vertices to the average of the $e ! \omega$-smallest diagrams obtained from $D$ by choosing any order on the $H_{\mathbb{Q}^{-}}$colored external vertices, and by declaring them to be larger than the $\omega$-vertex. One can prove that $k$ is an isomorphism, similarly to the proof in $\S 3.1$ that $\chi: \mathcal{A} \rightarrow \mathcal{A}^{<}$is an isomorphism.

Next, we define the linear map

$$
\lambda:=\chi^{-1} \circ \lambda^{<} \circ k: \check{I} \stackrel{k}{\longrightarrow} \check{I}^{<} \stackrel{\lambda^{<}}{\longrightarrow} I^{<} \stackrel{\chi^{-1}}{\longrightarrow} I .
$$

Since $\lambda$ is surjective, it is enough to prove that, for all $\omega$-diagram with only one $\omega$-vertex

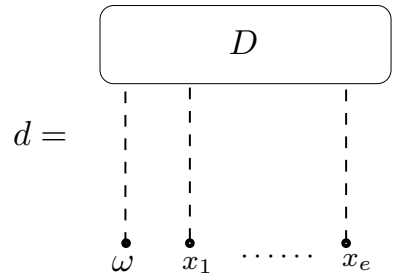


$\lambda(d)$ is given by formula (7.9). Thus, we have to compute

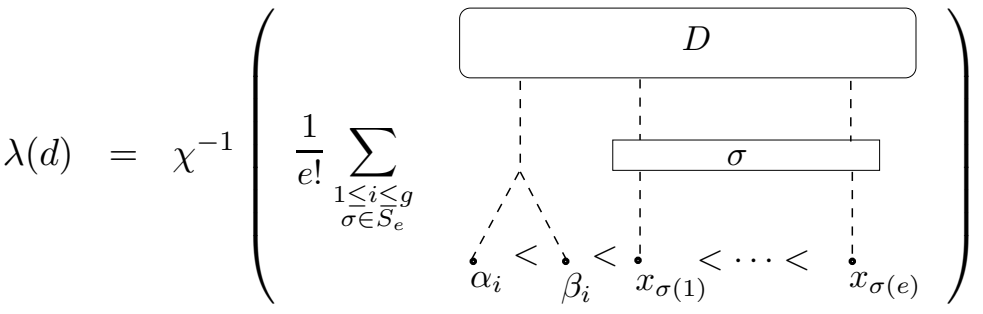

$$
\begin{aligned}
& =t_{0}+t_{1}+t_{2}+\cdot \cdot \cdot \cdot
\end{aligned}
$$

where $t_{p}$ is the term involving $p$ simultaneous contractions in the formula (3.1) that describes $\chi^{-1}$.

The term $t_{0}$ is $d$ itself, seen as an element of $\mathcal{A}$ using the rule (7.10). The term $t_{1}$ involves three types of contraction:

(1) The $\alpha_{i}$-colored vertex is contracted with the $\beta_{i}$-colored vertex: This gives a diagram with a loop edge, which is trivial by the AS relation.

(2) The $\alpha_{i}$-colored vertex, or the $\beta_{i}$-colored vertex, is contracted with an $x_{\sigma(j)^{-}}$ colored vertex. The sum of all these contractions (over $i=1, \ldots, g$ and $j=$ $1, \ldots, e)$ gives the following linear combination of diagrams:

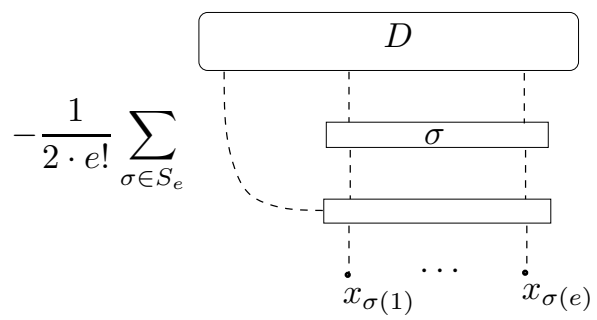

The generic term of this sum can be transformed by the IHX and AS relations to a diagram with a loop edge, and so is trivial.

(3) An $x_{\sigma(j)}$-colored vertex is contracted with an $x_{\sigma(i)}$-colored vertex: Such a term will cancel with another one of the same kind since $\omega$ is skew-symmetric.

So, $t_{1}$ is trivial. As for the term $t_{2}$, the above arguments show that it is enough to consider the contraction of the $\alpha_{i}$-colored vertex with an $x_{\sigma(j)}$-colored vertex together with the contraction of the $\beta_{i}$-colored vertex with an $x_{\sigma(k)}$-colored vertex (where $j \neq k$ and $j, k \in\{1, \ldots, e\})$. The sum of all these contractions gives the second term of the formula (7.9). Finally, the higher terms $t_{p}$ are trivial since a $p$-fold contraction with $p \geq 2$ should involve at least the contraction of an $x_{\sigma(j)}$-colored vertex with an $x_{\sigma(i)}$-colored vertex.

7.6. Algebraic description of $\operatorname{Gr} \mathbf{c} \otimes \mathbb{Q}$. In this subsection, we prove analogues of Theorems 1.4 and 1.5 for the closed surface $\Sigma_{g}$. In this case, the first Johnson homomorphism is a map

$$
\tau_{1}: \mathcal{I}_{g} \longrightarrow \Lambda^{3} H / H
$$

where $H$ embeds into $\Lambda^{3} H$ by $h \mapsto h \wedge \omega$. It is induced by the first Johnson homomorphism of the boundary case:

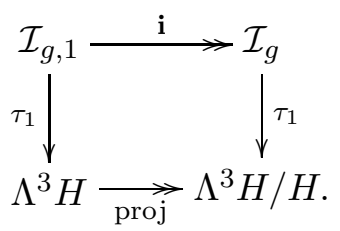


The homomorphism $\tau_{1}$ induces an isomorphism between $\operatorname{Gr}_{1}^{\Gamma} \mathcal{I}_{g} \otimes \mathbb{Q}$ and $\Lambda^{3} H_{\mathbb{Q}} / H_{\mathbb{Q}}[16]$, hence a Lie algebra epimorphism

$$
J: \operatorname{Lie}\left(\Lambda^{3} H_{\mathbb{Q}} / H_{\mathbb{Q}}\right) \longrightarrow \operatorname{Gr}^{\Gamma} \mathcal{I}_{g} \otimes \mathbb{Q} .
$$

Define $\mathrm{R}\left(\mathcal{I}_{g}\right):=\operatorname{Ker}(J)$. Hain's Theorem 1.1 holds in the closed case as well. See [14] where the following result is also proved:

Proposition 7.11 (Hain). If $g \geq 3$, then the $\operatorname{Sp}\left(H_{\mathbb{Q}}\right)$-module of quadratic relations $\mathrm{R}_{2}\left(\mathcal{I}_{g}\right)$ is spanned by the class of $r_{1}$, where $r_{1} \in \operatorname{Lie}_{2}\left(\Lambda^{3} H_{\mathbb{Q}}\right)$ has been defined in $\S 5.2$.

As an analogue of Theorem 1.4 in the closed case, we obtain the following statement:

Theorem 7.12. If $g \geq 3$, then the following diagram in the category of graded Lie algebras with $\mathrm{Sp}\left(H_{\mathbb{Q}}\right)$-actions is commutative:

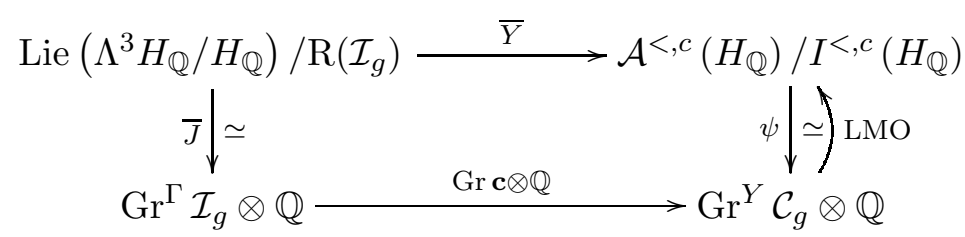

Here, $\bar{Y}$ is induced by the Lie algebra map $Y$ : Lie $\left(\Lambda^{3} H_{\mathbb{Q}} / H_{\mathbb{Q}}\right) \rightarrow \mathcal{A}^{<, c}\left(H_{\mathbb{Q}}\right) / I^{<, c}\left(H_{\mathbb{Q}}\right)$ which, in degree 1 , sends the class of $x \wedge y \wedge z$ to the class of the $Y$-graph $x^{y} z$.

Proof. The diagram commutes because its analogue for the surface $\Sigma_{g, 1}$ does.

Let us now consider the situation in degree 2. Again, it is convenient to switch from $\mathcal{A}^{<} / I^{<}$to $\mathcal{A} / I$. Thus, we consider the Lie bracket of $\mathcal{A}^{c} / I^{c}$ in degree $1+1$ :

$$
c_{2}:=[-,-]_{\star}: \Lambda^{2}\left(\mathcal{A}_{1}^{c} / I_{1}^{c}\right) \longrightarrow \mathcal{A}_{2}^{c} / I_{2}^{c} .
$$

According to Proposition 7.10 , the subspace $I_{2}^{c} \subset \mathcal{A}_{2}^{c}$ is generated by

$$
\int_{x}^{\omega}-\frac{\omega(x, y)}{4} \cdot \cdots, \quad \forall x, y \in H_{\mathbb{Q}}
$$

So, $I_{2}^{c}$ is contained in the even part $\mathcal{A}_{2, \mathrm{ev}}^{c}$.

Proposition 7.13. If $g \geq 3$, then we have

$$
\operatorname{Im}\left(c_{2}\right)=\mathcal{A}_{2, \mathrm{ev}}^{c} / I_{2}^{c} \quad\left(\simeq \Gamma_{0}+\Gamma_{2 \omega_{2}}\right) \quad \text { and } \quad \operatorname{Ker}\left(c_{2}\right)=\left\langle\left\{r_{1}\right\}\right\rangle_{\mathrm{Sp}\left(H_{\mathbb{Q}}\right)} .
$$

Proof. Proposition 5.5 and the commutative diagram

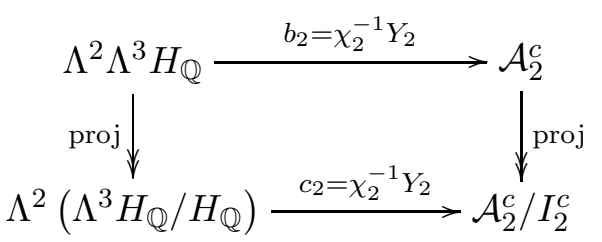


shows that the image of $c_{2}$ coincides with $\mathcal{A}_{2, \text { ev }}^{c} / I_{2}^{c}$. The irreducible decomposition of $\mathcal{A}_{2, \mathrm{ev}}^{c} / I_{2}^{c}$ is deduced from the decomposition of $\mathcal{A}_{2, \mathrm{ev}}^{c}$ :

\begin{tabular}{c|c} 
eigenvalue & eigenvector \\
\hline $2 \omega_{2}$ & $\left\{\begin{array}{c}\alpha_{2} \alpha_{1} \\
\vdots \\
\alpha_{1} \alpha_{2}\end{array}\right\}$ \\
\hline 0 & $\left\{\begin{array}{l}\alpha_{\omega} \quad \omega \\
\omega\end{array}\right\}=\left\{\frac{g}{4} \cdot \cdots\right.$
\end{tabular}

Let us now identify the kernel of $c_{2}$. First, we observe that

$$
\operatorname{Ker}\left(\mathcal{A}_{2, \mathrm{ev}}^{c} \rightarrow \mathcal{A}_{2, \mathrm{ev}}^{c} / I_{2}^{c}\right)=\left\langle\hat{\alpha}_{\alpha_{1}}^{\omega} \alpha_{2}, \omega, \omega-\frac{g}{4} \cdot\right\rangle_{\mathrm{Sp}\left(H_{\mathbb{Q}}\right)} .
$$

Moreover, the proof of Proposition 5.5 shows that

$$
\begin{aligned}
& b_{2}\left(\begin{array}{cccc}
\beta_{1} & & \\
- & & \wedge & \alpha_{1}
\end{array}\right)={\stackrel{\alpha}{\alpha_{1}}}_{\alpha_{2}}^{\omega}, \\
& \text { and } \quad b_{2}\left(\frac{1}{g-1} \sum_{i=1}^{g} \omega \quad \alpha_{i} \wedge{ }_{\omega} \beta_{i}\right)=\omega_{\omega} \omega_{\omega}-\frac{g}{4} \cdot
\end{aligned}
$$

Thus,

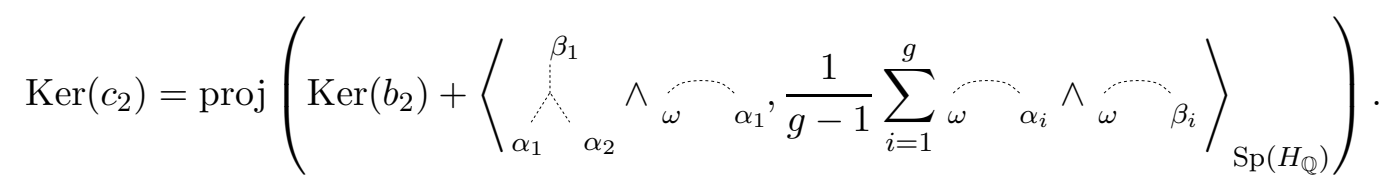

We conclude with Lemma 5.6 that $\operatorname{Ker}\left(c_{2}\right)$ is $\operatorname{Sp}\left(H_{\mathbb{Q}}\right)$-spanned by $\left\{r_{1}\right\}$.

Finally, we have the following commutative diagram:

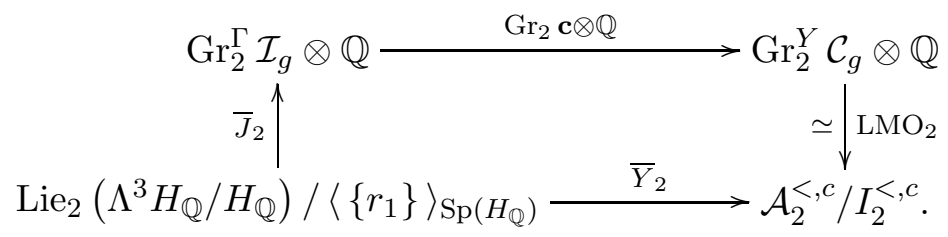

The previous proposition shows that $\bar{Y}_{2}$ is injective, which implies that $\bar{J}_{2}$ is injective (hence Hain's Proposition 7.11). We conclude that

$$
\operatorname{Ker}\left(Y_{2}\right)=R_{2}\left(\mathcal{I}_{g}\right)
$$

which is the analogue of Theorem 1.5 in the closed case. Thus, the map Gr $\mathbf{c} \otimes \mathbb{Q}$ : $\operatorname{Gr}^{\Gamma} \mathcal{I}_{g} \otimes \mathbb{Q} \rightarrow \operatorname{Gr}^{Y} \mathcal{C}_{g} \otimes \mathbb{Q}$ is injective in degree 2 .

\section{REMARKS AND QUESTIONS}

In this section, we assume $g \geq 6$ for simplicity. Some statements can be adapted to more general cases. Also, we consider only the surfaces with one boundary component, though most of the arguments below generalize to closed surfaces. 
8.1. The Lie subalgebra $a\left(H_{\mathbb{Q}}\right) \subset \mathcal{A}^{c}\left(H_{\mathbb{Q}}\right)$. Let $a\left(H_{\mathbb{Q}}\right)$ be the Lie subalgebra of $\mathcal{A}^{c}\left(H_{\mathbb{Q}}\right)$ generated by its degree 1 part $\mathcal{A}_{1}^{c}\left(H_{\mathbb{Q}}\right) \simeq \Lambda^{3} H_{\mathbb{Q}}$.

Question 8.1. Is the graded Lie algebra $a\left(H_{\mathbb{Q}}\right)$ quadratically presented?

According to Lemma 5.6, $a\left(H_{\mathbb{Q}}\right)$ has a quadratic presentation if and only if the kernel of the Lie algebra map

$$
\chi^{-1} \circ Y: \operatorname{Lie}\left(\Lambda^{3} H_{\mathbb{Q}}\right) \longrightarrow \mathcal{A}^{c}\left(H_{\mathbb{Q}}\right), \quad x \wedge y \wedge z \longmapsto{ }_{x}^{y}
$$

is generated as an ideal by $\left\langle r_{1}, r_{2}\right\rangle_{\mathrm{Sp}\left(H_{\mathbb{Q}}\right)}$.

By Theorem 1.4, Question 8.1 is equivalent to the fact that the Torelli Lie algebra is quadratically presented (i.e. the second part of Hain's Theorem 1.1) and the injectivity of $\mathrm{Gr} \mathbf{c} \otimes \mathbb{Q}: \mathrm{Gr}^{\Gamma} \mathcal{I}_{g, 1} \otimes \mathbb{Q} \longrightarrow \operatorname{Gr}^{Y} \mathcal{C}_{g, 1} \otimes \mathbb{Q}$.

Another very interesting problem would be to characterize the elements of $\mathcal{A}^{c}\left(H_{\mathbb{Q}}\right)$ belonging to $a\left(H_{\mathbb{Q}}\right)$. Recall from (3.6) that $a\left(H_{\mathbb{Q}}\right)$ is contained in $\mathcal{A}_{*, \text { ev }}^{c}\left(H_{\mathbb{Q}}\right)$.

8.2. Johnson homomorphisms and symplectic tree diagrams. Let us explain how the questions asked in $\S 8.1$ connect to problems about Johnson homomorphisms. Let

$$
\mathcal{I}_{g, 1}=\mathcal{I}_{g, 1}[1] \supset \mathcal{I}_{g, 1}[2] \supset \mathcal{I}_{g, 1}[3] \supset \cdots
$$

be the Johnson filtration of the Torelli group [17], defined by

$$
\mathcal{I}_{g, 1}[i]:=\operatorname{Ker}\left(\mathcal{I}_{g, 1} \longrightarrow \operatorname{Aut}\left(\pi_{1} \Sigma_{g, 1} / \Gamma_{i+1}\left(\pi_{1} \Sigma_{g, 1}\right)\right)\right) \quad \text { for } i \geq 1 .
$$

It is proved in [29] that

$$
\left[\mathcal{I}_{g, 1}[k], \mathcal{I}_{g, 1}[l]\right] \subset \mathcal{I}_{g, 1}[k+l] \quad \forall k, l \geq 1
$$

hence a Lie bracket on the graded Abelian group $\mathrm{Gr}^{[-]} \mathcal{I}_{g, 1}$. Also, $\operatorname{id}_{\mathcal{I}_{g, 1}}$ induces a Lie algebra homomorphism

$$
j: \mathrm{Gr}^{\Gamma} \mathcal{I}_{g, 1} \otimes \mathbb{Q} \longrightarrow \mathrm{Gr}^{[-]} \mathcal{I}_{g, 1} \otimes \mathbb{Q} .
$$

The following problem was proposed by Morita (in the case of closed surfaces):

Question 8.2 (See [30, Problem 6.2]). Is the map $j$ injective in degree $\neq 2$ ?

The Johnson homomorphisms (tensored by $\mathbb{Q})^{6}$ induce a Lie algebra monomorphism

$$
\tau: \mathrm{Gr}^{[-]} \mathcal{I}_{g, 1} \otimes \mathbb{Q} \longrightarrow \mathcal{A}^{c, t}\left(H_{\mathbb{Q}}\right),
$$

where

$$
\mathcal{A}^{c, t}\left(H_{\mathbb{Q}}\right):=\mathcal{A}^{c}\left(H_{\mathbb{Q}}\right) /(\text { diagrams with loops })
$$

consists of symplectic tree diagrams. The Lie bracket on $\mathcal{A}^{c, t}\left(H_{\mathbb{Q}}\right)$, introduced by Kontsevich [19] and Morita [29], coincides with the tree reduction of the Lie bracket $[-,-]_{\star}$ on $\mathcal{A}^{c}\left(H_{\mathbb{Q}}\right)$ defined in $\S 3.1$.

As announced in [13] and proved in $[8,11]$, the Johnson homomorphisms naturally extend to the monoid of homology cylinders, and one can describe these extensions using symplectic tree diagrams. Moreover, we have

$$
Y_{i} \mathcal{C}_{g, 1} \subset \mathcal{C}_{g, 1}[i] \quad \forall i \geq 1 .
$$

\footnotetext{
${ }^{6}$ The definition of Johnson homomorphisms needs several sign conventions. To be specific, the homomorphism $\tau_{i}: \mathcal{I}_{g, 1}[i] \rightarrow \mathcal{A}_{i}^{c, t}\left(H_{\mathbb{Q}}\right)$ that we are considering here is the homomorphism $\tau_{i}:$ $\mathcal{I}_{g, 1}[i] \rightarrow \mathcal{A}_{i}^{Y, c, t}\left(\lfloor g\rceil^{+} \cup\lfloor g\rceil^{-}\right)$as defined in [4], composed with the tree-reduction of the isomorphism $\chi^{-1} s \varphi: \mathcal{A}^{Y, c}\left(\lfloor g\rceil^{+} \cup\lfloor g\rceil^{-}\right) \rightarrow \mathcal{A}^{c}\left(H_{\mathbb{Q}}\right)$.
} 
So, $\operatorname{id}_{\mathcal{C}_{g, 1}}$ induces a Lie algebra homomorphism

$$
k: \operatorname{Gr}^{Y} \mathcal{C}_{g, 1} \otimes \mathbb{Q} \longrightarrow \operatorname{Gr}^{[-]} \mathcal{C}_{g, 1} \otimes \mathbb{Q} .
$$

Then, the commutative square (1.7) can be expanded to the commutative cube drawn in Figure 8.1. There, $r_{3} \in \operatorname{Lie}_{2}\left(\Lambda^{3} H_{\mathbb{Q}}\right) \simeq \Lambda^{2} \mathcal{A}_{1}^{c}\left(H_{\mathbb{Q}}\right)$ is a pre-image of the graph by $[-,-]_{\star}$. Commutativity of the rightmost square follows from the domination of the Johnson homomorphisms by the LMO invariant [4].

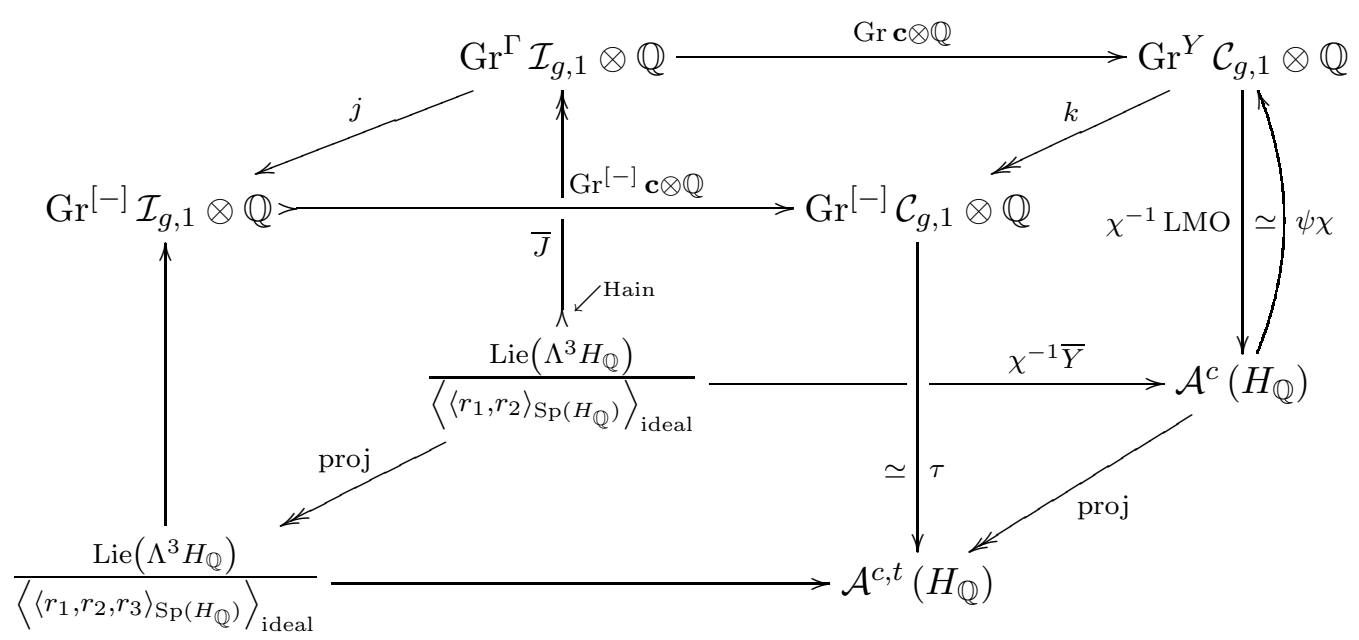

FiguRE 8.1. A cube of graded Lie algebras with $\operatorname{Sp}\left(H_{\mathbb{Q}}\right)$-actions.

Let $a^{t}\left(H_{\mathbb{Q}}\right)$ be the Lie subalgebra of $\mathcal{A}^{c, t}\left(H_{\mathbb{Q}}\right)$ generated by its degree 1 part $\mathcal{A}_{1}^{c, t}\left(H_{\mathbb{Q}}\right) \simeq$ $\Lambda^{3} H_{\mathbb{Q}}$. Question 8.1 has the following analogue at the "tree" level.

Question 8.3. Is the graded Lie algebra $a^{t}\left(H_{\mathbb{Q}}\right)$ quadratically presented?

By Lemma 5.6, $a^{t}\left(H_{\mathbb{Q}}\right)$ has a quadratic presentation if and only if the kernel of the Lie algebra map

$$
\operatorname{Lie}\left(\Lambda^{3} H_{\mathbb{Q}}\right) \longrightarrow \mathcal{A}^{c, t}\left(H_{\mathbb{Q}}\right), \quad x \wedge y \wedge z \longmapsto Y_{x}^{y}
$$

is generated as an ideal by $\left\langle r_{1}, r_{2}, r_{3}\right\rangle_{\mathrm{Sp}\left(H_{\mathbb{Q}}\right)}$.

The Lie subalgebra $a^{t}\left(H_{\mathbb{Q}}\right)$ is equal to the image of the Johnson homomorphisms:

$$
a^{t}\left(H_{\mathbb{Q}}\right)=\operatorname{Im}\left(\tau: \mathrm{Gr}^{[-]} \mathcal{I}_{g, 1} \otimes \mathbb{Q} \longrightarrow \mathcal{A}^{c, t}\left(H_{\mathbb{Q}}\right)\right) .
$$

We deduce that $a\left(H_{\mathbb{Q}}\right)$ is strictly contained in $\mathcal{A}_{*, \text { ev }}^{c}\left(H_{\mathbb{Q}}\right)$, since $\tau$ is not surjective, as was shown by Morita [29].

Remark 8.4. We can verify Question 8.1 in degree 3, with a long computation by hand. Since the projection $\mathcal{A}_{3, \mathrm{ev}}^{c}\left(H_{\mathbb{Q}}\right) \rightarrow \mathcal{A}_{3}^{c, t}\left(H_{\mathbb{Q}}\right)$ is bijective, it follows that Question 8.3 has a positive answer in degree 3 . This fact might be well known for experts (though it does not seem to have been proved or announced explicitly in the literature), since a version for closed surfaces is already proved by Morita [30, Proposition 6.3].

Moreover, we deduce that $r_{3}$ is central in the Lie algebra

$$
\text { Lie }\left(\Lambda^{3} H_{\mathbb{Q}}\right) /\left\langle\left\langle r_{1}, r_{2}\right\rangle_{\mathrm{Sp}\left(H_{\mathbb{Q}}\right)}\right\rangle_{\text {ideal }},
$$


so that the projection

$$
\operatorname{Lie}\left(\Lambda^{3} H_{\mathbb{Q}}\right) /\left\langle\left\langle r_{1}, r_{2}\right\rangle_{\operatorname{Sp}\left(H_{\mathbb{Q}}\right)}\right\rangle_{\text {ideal }} \longrightarrow \operatorname{Lie}\left(\Lambda^{3} H_{\mathbb{Q}}\right) /\left\langle\left\langle r_{1}, r_{2}, r_{3}\right\rangle_{\mathrm{Sp}\left(H_{\mathbb{Q}}\right)}\right\rangle_{\text {ideal }}
$$

is bijective in all degree $\neq 2$. Then, the commutative cube of Figure 8.1 shows that Question 8.3 implies Questions 8.1 and 8.2. Also, giving a positive answer to Question 8.3 by algebraic means would provide a new, algebraic proof for the second half of Hain's Theorem 1.1.

\section{Appendix A. On Malcev Lie algebras of Filtered Groups}

In this appendix, we define the "Malcev completion" and the "Malcev Lie algebra" of a filtered group. When the filtration is the lower central series, our definitions agree with the usual constructions of the Malcev completion and Malcev Lie algebra of a group. Our exposition is inspired by Quillen's appendix to his paper [34], which deals with the usual case. We fix a ground field $\mathbb{K}$ of characteristic 0 . We start by recalling some general definitions.

A filtration on a vector space $V$ is a descending chain of subspaces

$$
V=F_{0} V \supset F_{1} V \supset F_{2} V \supset \cdots .
$$

A complete vector space is a filtered vector space $V$ such that $V \stackrel{\simeq}{\longrightarrow} \lim _{i} V / F_{i} V$.

The complete tensor product $V \widehat{\otimes} W$ of two filtered vector spaces $V$ and $W$ is the completion of $V \otimes W$ with respect to the filtration

$$
F_{n}(V \otimes W):=\sum_{i+j=n} F_{i} V \otimes F_{j} W .
$$

The category of complete vector spaces endowed with $\widehat{\otimes}$ is a symmetric monoidal category. The unit object is $\mathbb{K}$ filtered by $\mathbb{K}=F_{0} \mathbb{K} \supset F_{1} \mathbb{K}=\{0\}$, and the transpose map $V \widehat{\otimes} W \rightarrow W \widehat{\otimes} V$ is the completion of the usual transpose map $V \otimes W \rightarrow W \otimes V$.

Definition A.1. A complete Hopf algebra is a Hopf algebra in the symmetric monoidal category of complete vector spaces.

Remark A.2. The reader is referred to [34, Appendix A] for a detailed treatment of complete Hopf algebras. It should be observed that Quillen's definition of a "complete Hopf algebra" $A$ is more restrictive than ours, since he requires the associated graded algebra to be generated by its degree 1 subspace. Consequently, some of the results of [34] do not directly generalize to our situation.

A filtration ${ }^{7}$ of a group $G$ is a descending chain of subgroups

$$
G=F_{1} G \supset F_{2} G \supset F_{3} G \supset \cdots
$$

such that $\left[F_{i} G, F_{j} G\right] \subset F_{i+j} G$. A filtration of a Lie algebra is defined in a similar way.

Example A.3. The lower central series of a group $G$ is a filtration of $G$, and similarly for a Lie algebra.

\footnotetext{
${ }^{7}$ Originally termed "N-series" by Lazard [20].
} 
In the sequel, we consider a filtered group $G$. Let $\mathbb{K}[G]$ be its group algebra, and let $I$ be the augmentation ideal of $\mathbb{K}[G]$. The given filtration on $G$ induces a filtration on the algebra $\mathbb{K}[G]$ by defining

$$
F_{i} \mathbb{K}[G]:=\left\langle\begin{array}{l|l}
\left(g_{1}-1\right) \cdots\left(g_{r}-1\right) & \begin{array}{l}
g_{1} \in F_{k_{1}} G, \ldots, g_{r} \in F_{k_{r}} G \\
k_{1}+\cdots+k_{r} \geq i
\end{array}
\end{array}\right\rangle_{\mathbb{K}} .
$$

Example A.4. If $G$ is filtered by the lower central series, then the filtration (A.1) is the $I$-adic filtration $\left(I^{k}\right)_{k \geq 0}$ of $\mathbb{K}[G]$.

The completion of $\mathbb{K}[G]$ with respect to the filtration (A.1) is denoted by

$$
\widehat{\mathbb{K}}[G]:=\lim _{i} \mathbb{K}[G] / F_{i} \mathbb{K}[G] .
$$

The filtration on $\mathbb{K}[G]$ induces a filtration on $\widehat{\mathbb{K}}[G]$ defined by

$$
F_{j} \widehat{\mathbb{K}}[G]:=\lim _{i \geq j} F_{j} \mathbb{K}[G] / F_{i} \mathbb{K}[G] .
$$

The group Hopf algebra structure on $\mathbb{K}[G]$ induces a cocommutative, complete Hopf algebra structure on $\widehat{\mathbb{K}}[G]$.

It follows that $\operatorname{Gr} \widehat{\mathbb{K}}[G] \simeq \operatorname{Gr} \mathbb{K}[G]$ is a graded cocommutative Hopf algebra. A result by Quillen describes it as the universal enveloping algebra of a graded Lie algebra. See [33] in the case of the lower central series and [24] in the general case:

Theorem A.5. Let $\operatorname{Gr} G$ be the graded Lie algebra over $\mathbb{Z}$ induced by the filtration of $G$, namely

$$
\operatorname{Gr} G=\bigoplus_{i \geq 1} F_{i} G / F_{i+1} G
$$

with Lie bracket induced by commutator in $G$. Then, there is a graded Hopf algebra isomorphism

$$
\theta: U(\mathrm{Gr} G \otimes \mathbb{K}) \stackrel{\simeq}{\longrightarrow} \mathrm{Gr} \mathbb{K}[G]
$$

defined by $\{g\} \otimes 1 \mapsto\{g-1\}$ for all $g \in F_{i} G$ and for all $i \geq 1$.

The complete vector space $\widehat{\mathbb{K}}[G]$ being a complete Hopf algebra, we can consider the primitive and the group-like elements of $\widehat{\mathbb{K}}[G]$.

Definition A.6. The Malcev completion of the filtered group $G$ is the group of grouplike elements of $\widehat{\mathbb{K}}[G]$

$$
\mathcal{G}(G):=\mathrm{G} \widehat{\mathbb{K}}[G]=\{x \in \widehat{\mathbb{K}}[G]: \widehat{\Delta} x=x \widehat{\otimes} x, x \neq 0\} .
$$

filtered by

$$
F_{i} \mathcal{G}(G):=\mathcal{G}(G) \cap\left(1+F_{i} \widehat{\mathbb{K}}[G]\right), \quad \forall i \geq 1 .
$$

The Malcev Lie algebra of $G$ is the Lie algebra of primitive elements of $\widehat{\mathbb{K}}[G]$

$$
\mathcal{P}(G):=\mathrm{P} \widehat{\mathbb{K}}[G]=\{x \in \widehat{\mathbb{K}}[G]: \widehat{\Delta} x=x \widehat{\otimes} 1+1 \widehat{\otimes} x\}
$$

filtered by

$$
F_{i} \mathcal{P}(G):=\mathcal{P}(G) \cap F_{i} \widehat{\mathbb{K}}[G], \quad \forall i \geq 1 .
$$

The canonical map $G \rightarrow \mathcal{G}(G)$ is denoted by $\iota$. It should be observed that $\iota$ is not necessarily injective. 
Example A.7. When $G$ is filtered by the lower central series, $\mathcal{G}(G)$ and $\mathcal{P}(G)$ are the usual notions of Malcev completion and Malcev Lie algebra respectively. This construction, via the completed group algebra, is due to Jennings [15] and Quillen [34].

As is always true in a complete Hopf algebra, the primitive and the group-like elements are in one-to-one correspondence by the exponential and logarithmic maps:

$$
\mathcal{G}(G) \subset 1+\widehat{I} \underset{\exp }{\rightleftarrows} \widehat{\log } \underset{I}{\rightleftarrows} \mathcal{P}(G) .
$$

Here, $\widehat{I} \subset \widehat{\mathbb{K}}[G]$ denotes the completion of the augmentation ideal $I \subset \mathbb{K}[G]$. The log and exp series converge on $1+\widehat{I}$ and $\widehat{I}$ respectively since $I^{k} \subset F_{k} \mathbb{K}[G]$ for all $k \geq 1$.

In the rest of this appendix, we extend two results, which are well-known for the lower central series, to arbitrary group filtrations. The first one is the following

Theorem A.8. The logarithmic map $\log \iota: G \rightarrow \mathcal{P}(G)$ induces a graded Lie algebra isomorphism:

$$
(\mathrm{Gr} \log \iota) \otimes \mathbb{K}: \operatorname{Gr} G \otimes \mathbb{K} \stackrel{\simeq}{\longrightarrow} \operatorname{Gr} \mathcal{P}(G) .
$$

Proof. First of all, $\log (\iota(g))=\log (1+(\iota g-1))=(\iota g-1)-(\iota g-1)^{2} / 2+\cdots$ belongs to $F_{i} \widehat{\mathbb{K}}[G]$ for all $g \in F_{i} G$ and for all $i \geq 1$. Thus, the map $\log \iota: G \rightarrow \mathcal{P}(G)$ preserves the filtration so that $\operatorname{Gr} \log \iota: \operatorname{Gr} G \rightarrow \operatorname{Gr} \mathcal{P}(G)$ is well-defined. The latter preserves the Lie brackets by the Baker-Campbell-Hausdorff formula. It remains to prove that the graded Lie algebra map $(\operatorname{Gr} \log \iota) \otimes \mathbb{K}: \operatorname{Gr} G \otimes \mathbb{K} \rightarrow \operatorname{Gr} \mathcal{P}(G)$ is bijective.

Quillen's isomorphism $\theta$ (Theorem A.5) gives a graded Lie algebra isomorphism at the level of primitive elements:

$$
\theta: \operatorname{Gr} G \otimes \mathbb{K} \stackrel{\simeq}{\longrightarrow} \mathrm{PGr} \mathbb{K}[G] .
$$

Moreover, there is the canonical Lie algebra homomorphism

$$
b: \operatorname{Gr} \mathcal{P}(G)=\operatorname{GrP} \widehat{\mathbb{K}}[G] \longrightarrow \mathrm{P} \mathrm{Gr} \widehat{\mathbb{K}}[G] \simeq \operatorname{P~Gr} \mathbb{K}[G] .
$$

The map $b$ is injective since the filtration that we are considering on $\mathcal{P}(G)$ is the restriction of the filtration on $\widehat{\mathbb{K}}[G]$. We conclude thanks to the following commutative triangle:

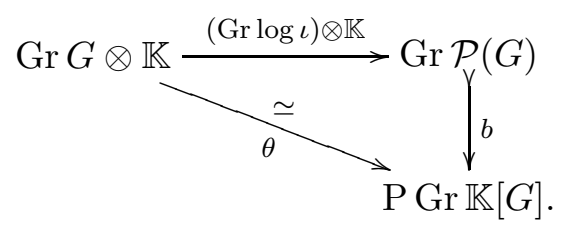

The usual form of Theorem A.8 is as follows:

Corollary A.9. If the group $G$ is filtered by the lower central series, then the logarithmic map $\log \iota: G \rightarrow \mathcal{P}(G)$ induces a graded Lie algebra isomorphism

$$
(\mathrm{Gr} \log \iota) \otimes \mathbb{K}: \operatorname{Gr} G \otimes \mathbb{K} \stackrel{\simeq}{\longrightarrow} \operatorname{Gr}^{\widehat{\Gamma}} \mathcal{P}(G),
$$

where $\widehat{\Gamma}$ is the complete lower central series ${ }^{8}$ of $\mathcal{P}(G)$.

\footnotetext{
${ }^{8}$ The complete lower central series of a complete Lie algebra $L$ is the filtration $\widehat{\Gamma}$ whose $i$-th term $\widehat{\Gamma}_{i} L$ is the closure of $\Gamma_{i} L$ in $L$.
} 
Proof of Corollary A.9. The Baker-Campbell-Hausdorff formula shows that the map $\log \iota: G \rightarrow \mathcal{P}(G)$ sends the lower central series of $G$ to the complete lower central series of $\mathcal{P}(G)$, so that $\operatorname{Gr} \log \iota: \operatorname{Gr} G \rightarrow \operatorname{Gr}^{\widehat{\Gamma}} \mathcal{P}(G)$ is well-defined. The same formula shows that $\operatorname{Gr} \log \iota$ preserves the Lie brackets.

Since $\Gamma_{n} \mathcal{P}(G) \subset F_{n} \mathcal{P}(G)$, we have $\widehat{\Gamma}_{n} \mathcal{P}(G) \subset F_{n} \mathcal{P}(G)$ hence a canonical map $c$ : $\operatorname{Gr}^{\widehat{\Gamma}} \mathcal{P}(G) \rightarrow \operatorname{Gr} \mathcal{P}(G)$. The commutative triangle

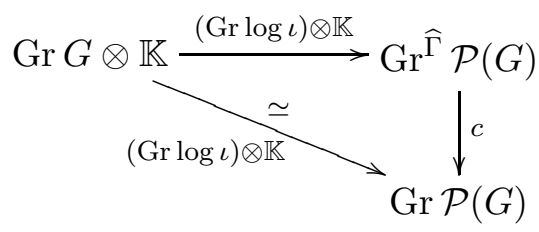

shows that $c$ is surjective. So, we have

$$
F_{n} \mathcal{P}(G) \subset\left(\widehat{\Gamma}_{n} \mathcal{P}(G)+F_{n+1} \mathcal{P}(G)\right), \quad \forall n \geq 1 .
$$

We deduce that $F_{n} \mathcal{P}(G)$ is contained in the closure of $\widehat{\Gamma}_{n} \mathcal{P}(G)$, so that $F_{n} \mathcal{P}(G)=$ $\widehat{\Gamma}_{n} \mathcal{P}(G)$. The conclusion follows.

To state the second result, we shall recall that, classically, the Malcev completion of a nilpotent group refers to its uniquely divisible closure. A uniquely divisible closure of a nilpotent group $N$ is a pair $(D, i)$, where

- $D$ is nilpotent and is uniquely divisible: $\forall y \in D, \forall k \geq 1, \exists ! x \in D, x^{k}=y$,

- $i: N \rightarrow D$ is a group homomorphism whose kernel is the torsion subgroup of $N$,

- $\forall x \in D, \exists k \geq 1, x^{k} \in i(N)$.

Malcev proved that the uniquely divisible closure of a nilpotent group $N$ exists and is essentially unique (see [18] for instance): Let us denote it by $N \otimes \mathbb{Q}$. As proved by Jennings in the finitely generated case [15] and by Quillen in general [34], the group $\mathcal{G}(N)$, where $N$ is filtered by the lower central series and where $\mathbb{K}=\mathbb{Q}$, is a realization of $N \otimes \mathbb{Q}$. This fact can be generalized as follows:

Theorem A.10. If the ground field is $\mathbb{K}=\mathbb{Q}$, then the canonical map $\iota: G \rightarrow \mathcal{G}(G)$ induces a group isomorphism

$$
\iota: \lim _{i}\left(\left(G / F_{i} G\right) \otimes \mathbb{Q}\right) \stackrel{\simeq}{\longrightarrow} \mathcal{G}(G) .
$$

Proof. For all $g \in G, \iota(g) \in \mathcal{G}(G)$ belongs to $1+F_{i} \widehat{\mathbb{Q}}[G]$ if and only if $g$ belongs to $1+F_{i} \mathbb{Q}[G]$. Consider the " $i$-th dimension subgroup" with coefficients in $\mathbb{Q}$ of the filtered group $G$, namely

$$
D_{i}(G):=\left\{g \in G \text { : the image of } g \text { in } \mathbb{Q}[G] \text { belongs to } 1+F_{i} \mathbb{Q}[G]\right\} .
$$

We can also consider the radical closure of $F_{i} G$ in $G$ :

$$
\sqrt{F_{i} G}:=\left\{g \in G: \exists k \geq 1, g^{k} \in F_{i} G\right\} .
$$

A classical result by Malcev asserts that $D_{i}(G)=\sqrt{F_{i} G}$ in the case of the lower central series (see [32] for instance), and it can be generalized to arbitrary filtrations (see [24]). Thus, the homomorphism $\iota: G \rightarrow \mathcal{G}(G)$ induces a group monomorphism

$$
\iota: G / \sqrt{F_{i} G} \longrightarrow \mathcal{G}(G) / F_{i} \mathcal{G}(G)
$$

for all $i \geq 1$. 
Next, we observe several properties for the group $\mathcal{G}(G) / F_{i} \mathcal{G}(G)$. First, it is nilpotent (being the quotient of a group by a term of a filtration). Second, it is torsion-free: Let $x \in \mathcal{G}(G)$ and $n \geq 1$ be such that $x^{n} \in F_{i} \mathcal{G}(G)$; setting $y:=\log (x)$, we deduce that $n y \in$ $F_{i} \widehat{\mathbb{Q}}[G]$ or, equivalently, that $y \in F_{i} \widehat{\mathbb{Q}}[G]$; we conclude that $x=\exp (y) \in\left(1+F_{i} \widehat{\mathbb{Q}}[G]\right)$. Third, the group $\mathcal{G}(G) / F_{i} \mathcal{G}(G)$ is divisible since $\mathcal{G}(G)=\exp \mathcal{P}(G)$ is.

Therefore, $\mathcal{G}(G) / F_{i} \mathcal{G}(G)$ is a uniquely divisible nilpotent group. To show that it the uniquely divisible closure of $G / \sqrt{F_{i} G}$ via (A.2), we prove

$$
\forall x \in \mathcal{G}(G), \exists k \geq 1, x^{k} \in \iota(G) \cdot F_{i} \mathcal{G}(G)
$$

by induction on $i \geq 1$. Thus, we consider an $x \in \mathcal{G}(G)$ satisfying that there exists $k \geq 1$, $g \in G$ and $y \in F_{i-1} \mathcal{G}(G)$ such that $x^{k}=\iota(g) y$. According to Theorem A.8, the map

$$
(\mathrm{Gr} G) \otimes \mathbb{Q} \longrightarrow \operatorname{Gr} \mathcal{G}(G)
$$

defined by $\{h\} \otimes q \mapsto\{\exp (q \log (\iota h))\}$ for all $h \in F_{j} G, j \geq 1$ and $q \in \mathbb{Q}$, is a bijection. We deduce that there exists $n \geq 1$ and $h \in G$ such that $y^{n}=\iota(h)$ modulo $F_{i} \mathcal{G}(G)$. Since $y \in F_{i-1} \mathcal{G}(G)$ is central modulo $F_{i} \mathcal{G}(G)$, we have that $x^{k n}=\iota(g)^{n} y^{n}=\iota\left(g^{n} h\right)$ modulo $F_{i} \mathcal{G}(G)$. This proves claim (A.3).

Thus, (A.2) induces a group isomorphism

$$
\iota:\left(G / \sqrt{F_{i} G}\right) \otimes \mathbb{Q} \longrightarrow \mathcal{G}(G) / F_{i} \mathcal{G}(G)
$$

defined by $\{x\}^{1 / n} \mapsto\left\{\exp \left(\frac{1}{n} \log (\iota x)\right)\right\}$ for all $x \in G$ and $n \geq 1$. Passing to the inverse limit, we obtain an isomorphism

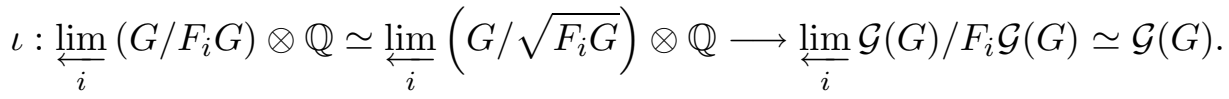

\section{REFERENCES}

[1] M. Asada, H. Nakamura, On graded quotient modules of mapping class groups of surfaces, Israel J. Math. 90 (1995) 93-113.

[2] D. Bar-Natan, S. Garoufalidis, L. Rozansky, D. Thurston, The Aarhus integral of rational homology 3-spheres I, Selecta Math. 8 (2002) 315-339.

[3] F. Bayen, M. Flato, C. Fronsdal, A. Lichnerowicz, D. Sternheimer, Deformation theory and quantization. I \& II., Ann. Physics 111 (1978) 61-151.

[4] D. Cheptea, K. Habiro, G. Massuyeau, A functorial LMO invariant for Lagrangian cobordisms, preprint arXiv:math/0701277, 2007.

[5] D. Cheptea, T. T. Q. Le, A TQFT associated to the LMO invariant of three-dimensional manifolds, Comm. Math. Phys. 272 (2007) 601-634.

[6] W. Fulton, J. Harris, Representation theory: A first course, Graduate Texts in Mathematics 129, Springer, Berlin, 1991.

[7] S. Garoufalidis, M. Goussarov, M. Polyak, Calculus of clovers and finite type invariants of 3manifolds, Geom. Topol. 5 (2001) 75-108.

[8] S. Garoufalidis, J. Levine, Tree-level invariants of 3-manifolds, Massey products and the Johnson homomorphism, Graphs and patterns in mathematics and theoretical physics, 173-203, Proc. Sympos. Pure Math. 73, Amer. Math. Soc., Providence, RI, 2005.

[9] M. Goussarov, Finite type invariants and n-equivalence of 3-manifolds, Compt. Rend. Acad. Sc. Paris 329 Série I (1999) 517-522.

[10] M. Goussarov, Knotted graphs and a geometrical technique of n-equivalence, St. Petersburg Math. J. 12 (2001) 569-604.

[11] N. Habegger, Milnor, Johnson and tree-level perturbative invariants, preprint, 2000.

[12] N. Habegger, C. Sorger, An infinitesimal presentation of the Torelli group of a surface with boundary, preprint, 2000.

[13] K. Habiro, Claspers and finite type invariants of links, Geom. Topol. 4 (2000) 1-83. 
[14] R. Hain, Infinitesimal presentations of the Torelli groups, J. Amer. Math. Soc. 10 (1997) 597-651.

[15] S. Jennings, The group ring of a class of infinite nilpotent groups, Canad. J. Math. 7 (1955) 169-187.

[16] D. Johnson, An Abelian quotient of the mapping class group $\mathcal{I}_{g}$, Math. Ann. 249 (1980) 225-242.

[17] D. Johnson, A survey of the Torelli group, Low-dimensional topology (San Francisco, Calif., 1981), 165-179, Contemp. Math. 20, Amer. Math. Soc., Providence, RI, 1983.

[18] M. Kargapolov, J. Merzljakov, Fundamentals of the theory of groups, Graduate Texts in Math. 62, Springer-Verlag, New York-Berlin, 1979.

[19] M. Kontsevich, Formal (non) commutative symplectic geometry, The Gelfand Mathematical Seminars, 1990-1992, 173-187, Birkhäuser Boston, Boston, MA, 1993.

[20] M. Lazard, Sur les groupes nilpotents et les anneaux de Lie, Ann. Sci. Ecole Norm. Sup. 71 (1954) 101-190.

[21] T. T. Q. Le, J. Murakami, T. Ohtsuki, On a universal perturbative invariant of 3-manifolds, Topology 37 (1998) 539-574.

[22] J. Levine, Homology cylinders: an enlargement of the mapping class group, Algebr. Geom. Topol. 1 (2001) 243-270.

[23] D. Littlewood, On invariant theory under restricted groups, Philos. Trans. Roy. Soc. London. Ser. A. 239 (1944) $387-417$.

[24] G. Massuyeau, Finite-type invariants of three-manifolds and the dimension subgroup problem, J. London Math. Soc. 75 (2007) 791-811.

[25] G. Massuyeau, J.-B. Meilhan, Characterization of $Y_{2}$-equivalence for homology cylinders, J. Knot Theory Ramifications 12 (2003) 493-522.

[26] G. Mess, The Torelli groups for genus 2 and 3 surfaces, Topology 31 (1992) 775-790.

[27] S. Morita, Casson's invariant for homology 3-spheres and characteristic classes of surface bundles, Topology 28 (1989) 305-323.

[28] S. Morita, On the structure of the Torelli group and the Casson invariant, Topology 30 (1991) 603-621.

[29] S. Morita, Abelian quotients of subgroups of the mapping class group of surfaces, Duke Math. J. 70 (1993) 699-726.

[30] S. Morita, Structure of the mapping class group of a surface: a survey and a prospect, Geom. Topol. Monog. 2 (1999) 349-406.

[31] J. Murakami, T. Ohtsuki, Topological quantum field theory for the universal quantum invariant, Comm. Math. Phys. 188 (1997) 501-520.

[32] I. Passi, Group rings and their augmentation ideals, Lecture Notes in Mathematics 715, SpringerVerlag, Berlin, 1979.

[33] D. Quillen, On the associated graded ring of a group ring, J. Algebra 10 (1968) 411-418.

[34] D. Quillen, Rational homotopy theory, Ann. of Math. 90 (1969) 205-295.

[35] T. Sakasai, The second Johnson homomorphism and the second rational cohomology of the Johnson kernel, preprint arXiv:math/0601314, 2006.

[36] T. Sakasai, Homology cylinders and the acyclic closure of a free group, Algebr. Geom. Topol. 6 (2006) 603-631.

Research Institute for Mathematical Sciences, Kyoto University, Kyoto 606-8502, Japan E-mail address: habiro@kurims.kyoto-u.ac.jp

Institut de Recherche Mathématique Avancée, Université Louis Pasteur-CNRS, 7 rue René Descartes, 67084 Strasbourg, France

E-mail address: massuyeau@math.u-strasbg.fr 